\title{
On the geometry of 2-categories and their classifying spaces *
}

\author{
M. BULLEJOS (bullejos@ugr.es) and A.M. CEGARRA \\ (cegarra@ugr.es) \\ Departamento de Álgebra, Universidad de Granada
}

\begin{abstract}
In this paper we prove that realizations of geometric nerves are classifying spaces for 2-categories. This result is particularized to strict monoidal categories and it is also used to obtain a generalization of Quillen's Theorem A.
\end{abstract}

Keywords: 2-category, monoidal category, simplicial space, nerve, classifying space, homotopy equivalence.

Math. Subjects Classification (2000): 18D05, 18D10, 55U40, 55P15, 55U10

\section{Introduction and Summary}

The construction of nerves and classifying spaces seeks to associate geometrical objects to categorical structures, in such a way that those objects should keep all the categorical structure information. This process of taking classifying spaces leads to the homotopy theory of those categorical structures, whose interest is well recognized; for example, let us recall that Quillen [13] defines a higher algebraic $K$-theory by taking homotopy groups of the classifying spaces of certain categories and also recall that classifying spaces of symmetric monoidal categories provide the most noteworthy examples of spaces with the extra structure required to define a $\Omega$-spectrum [18, 22].

It was Grothendieck [10] who first associated a simplicial set NC to a small category $\mathcal{C}$, calling this simplicial set the nerve of $\mathcal{C}$. The psimplices of $\mathrm{NC}$ are diagrams in $\mathcal{C}$ of the form $x_{0} \rightarrow x_{1} \rightarrow \ldots x_{p-1} \rightarrow x_{p}$. The classifying space of the category is the geometric realization [19] of its nerve, $\mathrm{BC}=|\mathrm{NC}|$. Later, Segal $[21]$ extended the realization process to simplicial (topological) spaces. He observed that if $\mathcal{C}$ is a topological category then $\mathrm{NC}$ is, in a natural way, a simplicial space and he defines the classifying space $\mathrm{BC}$ of a topological category $\mathcal{C}$ as the realization of the simplicial space NC. This general construction given by Segal provides, for instance, a definition of classifying spaces of 2-categories. A 2-category $\mathcal{C}$ is a category endowed with categorical hom-sets $\mathcal{C}(x, y)$, for any pair of objects, in such a way that the compositions $\mathcal{C}(x, y) \times$

* Supported by DGI:BFM2001-2886

(C) 2002 Kluwer Academic Publishers. Printed in the Netherlands. 
$\mathcal{C}(y, z) \rightarrow \mathcal{C}(x, z)$ are functors. By replacing the hom-categories $\mathcal{C}(x, y)$ by their classifying spaces $\mathrm{BC}(x, y)$, we obtain a topological category $\mathfrak{B C}$ (with discrete space of objects). Then, the classifying space $\mathrm{BC}$ of the 2-category $\mathcal{C}$ is defined as that of the topological category $\mathfrak{B C}$.

However, there is another convincing way of associating a space to a 2 -category $\mathcal{C}$. This way goes through what Duskin called [5] the geometric nerve $\Delta \mathcal{C}$ of the 2-category and it was developed, among others, by Street [23] and Duskin himself. This geometric nerve $\Delta \mathcal{C}$ is a simplicial set, which encodes the entire 2-categorical structure of $\mathcal{C}$, whose simplices have the following pleasing geometrical description. The vertices of $\Delta \mathcal{C}$ are the objects $x_{0}$ of $\mathcal{C}$, the 1 -simplices are the arrows $x_{0} \stackrel{x_{0,1}}{\longrightarrow} x_{1}$ of $\mathcal{C}$ and the 2 -simplices are triangles:

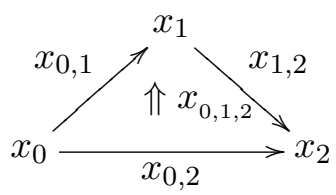

with $x_{0,1,2}: x_{0,2} \Rightarrow x_{1,2} x_{0,1}$ a deformation (2-cell) in $\mathcal{C}$. For $n \geq 3$, an $n$-simplex of $\Delta \mathcal{C}$ is a diagram in $\mathcal{C}$ with the shape of the 2 -skeleton of an oriented standard $n$-simplex,

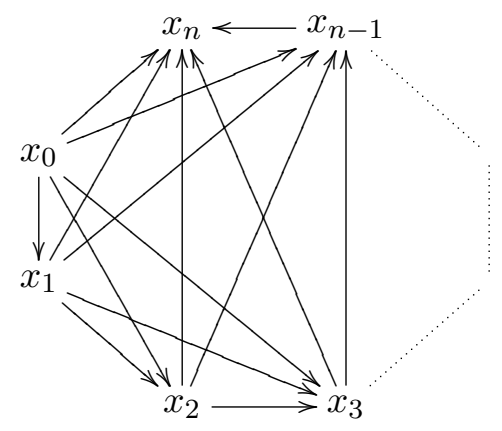

whose vertices are then objects $x_{0}, \ldots, x_{n}$, whose edges are arrows $x_{i} \stackrel{x_{i, j}}{\longrightarrow} x_{j}$, for $0 \leq i<j \leq n$, and whose faces are triangles:

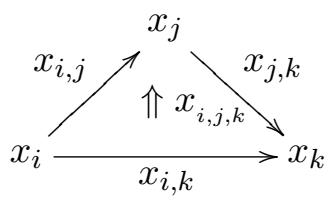


with $x_{i, j, k}: x_{i, k} \Rightarrow x_{j, k} x_{i, j}$ a deformation, for $0 \leq i<j<k \leq n$. These data are required to satisfy that each tetrahedron

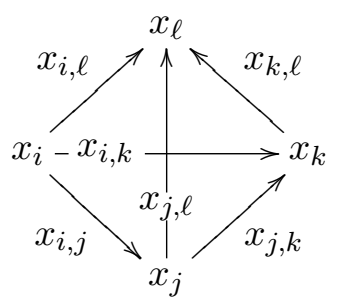

$$
\begin{aligned}
x_{i, j, k}: x_{i, k} & \Rightarrow x_{j, k} x_{i, j} \\
x_{i, j, \ell}: x_{i, \ell} & \Rightarrow x_{j, \ell} x_{i, j} \\
x_{i, k, \ell}: x_{i, \ell} & \Rightarrow x_{k, \ell} x_{i, k} \\
x_{j, k, \ell}: x_{j, \ell} & \Rightarrow x_{k, \ell} x_{j, k}
\end{aligned}
$$

for $0 \leq i<j<k<\ell \leq n$, is commutative in the sense that the following square of deformations

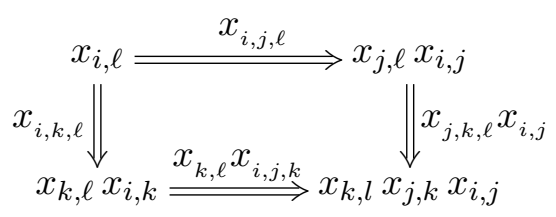

commutes. This simplicial set $\Delta \mathcal{C}$ becomes coskeletal in dimensions greater than 3 [23, Theorem 23].

Since we have two different spaces associated to a 2-category $\mathcal{C}$ (namely $\mathrm{BC}$ and $|\Delta \mathcal{C}|$ ) and both have shown their utility, it seems natural to compare them. To our knowledge, the only comparison that has been given is in the case in which all arrows and deformations of $\mathcal{C}$ are invertible, that is, when $\mathcal{C}$ is a 2 -groupoid. For this case, Moerdijk and Svensson [20] prove that the two classifying spaces associated as above to a 2 -groupoid $\mathcal{C}$ are homotopically equivalent. In their proof, the restriction on $\mathcal{C}$ is essential, so that it can not be translated to general 2-categories. The main purpose of this article is to prove the following:

THEOREM 1. For any 2-category $\mathcal{C}$ there is a natural homotopy equivalence

$$
\mathrm{BC} \stackrel{\sim}{\longrightarrow}|\Delta \mathcal{C}|
$$

The above Theorem 1 can be applied to strict monoidal categories when these are considered as 2-categories with only one object.

As an application we also prove Theorem 2 below, which is a generalization of Theorem A of Quillen [13] to 2-categories. In our theorem we replace the notion of homotopy fiber category of a functor by Gray's notion of homotopy fiber 2-category $y^{\prime} / / F$ of a 2 -functor $F: \mathcal{C} \rightarrow \mathcal{C}^{\prime}$ at an object $y^{\prime} \in \mathcal{C}^{\prime}[9]$ (see Section 1 for details). 
THEOREM 2. Let $F: \mathcal{C} \rightarrow \mathcal{C}^{\prime}$ be a 2-functor. If the classifying space $\mathrm{B}\left(y^{\prime} / / F\right)$ is contractible for every object $y^{\prime} \in \mathcal{C}^{\prime}$, then the induced map $\mathrm{B} F: \mathrm{BC} \rightarrow \mathrm{BC}^{\prime}$ is a homotopy equivalence.

This paper is organized into three sections. The first one is dedicated to stating some concepts, results and terminology we are going to use by reviewing definitions and basic facts concerning 2-categories, laxfunctors, nerves, realizations and classifying spaces. The material in this first section is pretty standard, except perhaps Proposition 1.1, so that an expert reader could skip this section. Section 2 is the main one and it includes the proof of Theorem 1. In Section 3 we prove Theorem 2.

\section{Preliminaries and Notations}

For the general background on 2-categories used in this paper we refer to $[2,15]$, and on simplicial sets to [16]. Throughout this paper all categories are assumed to be small.

2-CATEGories. A 2-category $\mathcal{C}$ is just a category enriched in the category of small categories. Then, $\mathcal{C}$ is a category in which the hom-set between any two objects $x, y \in \mathcal{C}$ is the set of objects of a category $\mathcal{C}(x, y)$, whose arrows are called deformations and are denoted $f: u \Rightarrow$ $v$. Moreover, the composition is a bifunctor $\mathcal{C}(x, y) \times \mathcal{C}(y, z) \rightarrow \mathcal{C}(x, z)$ which is associative and has identities $I d_{x} \in \mathcal{C}(x, x)$. This bifunctor produces compositions of arrows and deformations respectively, both denoted here by juxtaposition. On the other hand, composition in each category $\mathcal{C}(x, y)$ is denoted by "o". Besides, we will usually identify an arrow $w: y \rightarrow z$ with the identity deformation on $w$ in the category $\mathcal{C}(y, z)$, hence we write $w f: w u \Rightarrow w v$ for the composition of $f$ with the deformation identity on $w$.

The underlying category of a 2 -category $\mathcal{C}$ is the category obtained by leaving out the deformations of $\mathcal{C}$.

A 2-functor $F: \mathcal{C} \rightarrow \mathcal{C}^{\prime}$ between 2-categories is an enriched functor and so it takes objects, arrows and deformation in $\mathcal{C}$ to objects, arrows and deformations in $\mathcal{C}^{\prime}$ respectively, in such a way that all the 2-category

structure is preserved. Any 2-functor induces, by restriction, an ordinary functor between the corresponding underlying categories and another one $\mathcal{C}(x, y) \rightarrow \mathcal{C}^{\prime}(F(x), F(y))$, for any pair of objects $x, y \in \mathcal{C}$.

Given a 2-functor $F: \mathcal{C} \rightarrow \mathcal{C}^{\prime}$ and an object $y^{\prime} \in \mathcal{C}^{\prime}$ by the homotopy fiber 2-category $y^{\prime} / / F$ we mean Gray's lax comma category $\left\ulcorner y^{\prime}\right\urcorner / / F$ where $\left\ulcorner y^{\prime}\right\urcorner: \mathbf{1} \rightarrow \mathcal{C}^{\prime}$ is the "name of an object" 2-functor [9]. Its objects 
are pairs $\left(u^{\prime}, x\right)$ where $x \in \mathcal{C}$ is an object and $u^{\prime}: y^{\prime} \rightarrow F(x)$ is an arrow in $\mathcal{C}^{\prime}$. Its morphisms are also pairs

$$
\left(f^{\prime}, u\right):\left(u_{0}^{\prime}, x_{0}\right) \rightarrow\left(u_{1}^{\prime}, x_{1}\right)
$$

where $u: x_{0} \rightarrow x_{1}$ is an arrow in $\mathcal{C}$ and $f^{\prime}: u_{1}^{\prime} \Rightarrow F(u) u_{0}^{\prime}$ is a deformation in $\mathcal{C}^{\prime}$. Finally, its deformations

$$
f:\left(f_{0}^{\prime}, u_{0}\right) \Rightarrow\left(f_{1}^{\prime}, u_{1}\right)
$$

are deformations $f: u_{0} \Rightarrow u_{1}$ in $\mathcal{C}$ such that $f_{1}^{\prime}=F(f) u_{0}^{\prime} \circ f_{0}^{\prime}$.

LAX-FUNCTORS. Given a category $\mathcal{I}$ and a 2-category $\mathcal{C}$, a (normalized or strictly unitary) lax-functor $\mathbf{x}: \mathcal{I} \rightarrow \mathcal{C}$ consists of three maps which assign to each object $i \in \mathcal{I}$ an object $x_{i} \in \mathcal{C}$, to each arrow $\tau: i \rightarrow j$ in $\mathcal{I}$ an arrow $x_{\tau}: x_{i} \rightarrow x_{j}$ in $\mathcal{C}$ and to each pair of composable arrows $i \stackrel{\tau}{\longrightarrow} j \stackrel{\sigma}{\longrightarrow} k$ in $\mathcal{I}$ a deformation $x_{\sigma, \tau}: x_{\sigma \tau} \Rightarrow x_{\sigma} x_{\tau}$ in $\mathcal{C}$, respectively. These data are required to satisfy the following normalization and coherence conditions:

- for any object $i \in \mathcal{I}, x_{I d_{i}}=I d_{x_{i}}$,

- for any arrow $\tau: i \rightarrow j$ in $\mathcal{I}, x_{\tau, I d_{i}}=x_{\tau}=x_{I d_{j}, \tau}$,

- for any triple of composable arrows $i \stackrel{\gamma}{\longrightarrow} j \stackrel{\tau}{\longrightarrow} k \stackrel{\sigma}{\longrightarrow} \ell$ in $\mathcal{I}$,

$$
x_{\sigma, \tau} x_{\gamma} \circ x_{\sigma \tau, \gamma}=x_{\sigma} x_{\tau, \gamma} \circ x_{\sigma, \tau \gamma} .
$$

By a deformation $f: \mathbf{x} \Rightarrow \mathbf{y}$, between lax-functors $\mathbf{x}, \mathbf{y}: \mathcal{I} \rightarrow \mathcal{C}$, we mean a (normalized) lax-natural transformation. Then, $f$ consists of a pair of maps which assign to each object $i \in \mathcal{I}$ an arrow $f_{i}: x_{i} \rightarrow y_{i}$ in $\mathcal{C}$ and to each arrow $\tau: i \rightarrow j$ in $\mathcal{I}$ a deformation $f_{\tau}: f_{j} x_{\tau} \Rightarrow y_{\tau} f_{i}$ in $\mathcal{C}$, respectively. Such that:

- for any object $i \in \mathcal{I}, f_{I d_{i}}=f_{i}$,

- for any pair of composable arrows $i \stackrel{\tau}{\longrightarrow} j \stackrel{\sigma}{\longrightarrow} k$,

$$
y_{\sigma} f_{\tau} \circ f_{\sigma} x_{\tau} \circ f_{k} x_{\sigma, \tau}=y_{\sigma, \tau} f_{i} \circ f_{\sigma \tau} .
$$

When two lax-functors $\mathbf{x}$ and $\mathbf{y}$ coincide over a set of (base-) objects $\Theta \subseteq \mathcal{I}$, a deformation $f: \mathbf{x} \Rightarrow \mathbf{y}$ is qualified as "relative" to $\Theta$ whenever $f_{i}=I d_{x_{i}}$, for all objects $i \in \Theta$.

For a fixed (possibly empty) set of objects $\Theta$, lax-functors from $\mathcal{I}$ to $\mathcal{C}$ and deformations relative to $\Theta$ between them form a category that we write here as

$$
\underline{\ell \text { Func }}(\mathcal{I}, \mathcal{C})_{\operatorname{rel} \Theta}
$$


Note that lax-functors $\mathbf{x}: \mathcal{I} \rightarrow \mathcal{C}$ such that all deformations $x_{\sigma, \tau}$ are identities are just ordinary functors to the underlying category of $\mathcal{C}$, to which we refer simply as functors from $\mathcal{I}$ to $\mathcal{C}$. We write

$$
\underline{\operatorname{Func}}(\mathcal{I}, \mathcal{C})_{\operatorname{rel} \Theta}
$$

for the full subcategory of $\underline{\ell \text { Func }}(\mathcal{I}, \mathcal{C})_{\text {rel } \Theta}$ whose objects are the functors from $\mathcal{I}$ to $\mathcal{C}$. Observe that the coherence condition (2) for a deformation $f$ between functors $\mathbf{x}, \mathbf{y}: \mathcal{I} \rightarrow \mathcal{C}$ reduces to the equality

$$
f_{\sigma \tau}=y_{\sigma} f_{\tau} \circ f_{\sigma} x_{\tau} .
$$

Let us now replace category $\mathcal{I}$ by a (directed and reflexive multi) graph $\mathcal{G}$. By a morphism $\mathbf{x}: \mathcal{G} \rightarrow \mathcal{C}$ we mean a (directed and reflexive multi-) graph morphism from $\mathcal{G}$ to the underlying graph of the underlying category of $\mathcal{C}$. Then $\mathbf{x}$ consists of a pair of maps which assign an object $x_{i}$ to each vertex $i \in \mathcal{G}$ and an arrow $x_{\tau}: x_{i} \rightarrow x_{j}$ to each edge $\tau: i \rightarrow j$ in $\mathcal{G}$, respectively, such that the arrows associated to degenerated edges are identities, that is, $x_{I d_{i}}=I d_{x_{i}}$. Given a set $\Theta$ of vertices in $\mathcal{G}$ and two morphisms $\mathbf{x}, \mathbf{y}: \mathcal{G} \rightarrow \mathcal{C}$, by a deformation relative to $\Theta, f: x \Rightarrow y$, we mean a pair of maps that assign an arrow $f_{i}: x_{i} \rightarrow y_{i}$ in $\mathcal{C}$ to each vertex $i \in \mathcal{G}$ and a deformation $f_{\tau}: f_{j} x_{\tau} \Rightarrow y_{\tau} f_{i}$ to each edge $\tau: i \rightarrow j$, respectively, such that $f_{i}=I d_{x_{i}}$ for any vertex $i \in \Theta$ and $f_{I d_{j}}=I d_{f_{j}}$ for any vertex $j \in \mathcal{G}$. Morphisms form $\mathcal{G}$ to $\mathcal{C}$ and deformations relative to $\Theta$ form a category that we write as $\operatorname{Gph}(\mathcal{G}, \mathcal{C})_{\operatorname{rel} \Theta}$.

Let us now suppose that the category $\mathcal{I}=\mathcal{I}(\mathcal{G})$ is the free category generated by the graph $\mathcal{G}$. Then, any morphism $\mathbf{x}: \mathcal{G} \rightarrow \mathcal{C}$ has a unique extension to a functor $\widetilde{\mathbf{x}}: \mathcal{I} \rightarrow \mathcal{C}[15$, Theorem 1]; moreover, any deformation relative to $\Theta, f: \mathbf{x} \Rightarrow \mathbf{y}$, between morphisms has a unique extension to a deformation relative to $\Theta, \widetilde{f}: \widetilde{\mathbf{x}} \Rightarrow \widetilde{\mathbf{y}}$, between the corresponding functors. On a string $i_{0} \stackrel{\sigma_{1}}{\longrightarrow} i_{1} \stackrel{\sigma_{2}}{\longrightarrow} i_{2} \ldots i_{n-1} \stackrel{\sigma_{n}}{\longrightarrow} i_{n}$ of edges in $\mathcal{G}$, the deformation $\widetilde{f}$ is inductively defined, by using (3), by the formula

$$
\widetilde{f}_{\sigma_{n} \cdots \sigma_{1}}=y_{\sigma_{n}} \tilde{f}_{\sigma_{n-1} \cdots \sigma_{1}} \circ f_{\sigma_{n}} \widetilde{x}_{\sigma_{n-1} \cdots \sigma_{1}} .
$$

Therefore, by restriction, we have an isomorphism of categories

$$
\underline{\operatorname{Func}}(\mathcal{I}(\mathcal{G}), \mathcal{C})_{\mathrm{rel} \Theta} \cong \operatorname{Gph}(\mathcal{G}, \mathcal{C})_{\operatorname{rel} \Theta}
$$

Bellow we shall also comment on lax-functors from free categories.

PROPOSITION 1.1. Let $\mathcal{I}$ be the free category generated by a graph $\mathcal{G}$ and let $\Theta$ be a subset of objects of $\mathcal{I}$. Then, for any 2-category $\mathcal{C}$, the subcategory $\underline{\operatorname{Func}}(\mathcal{I}, \mathcal{C})_{\mathrm{rel} \Theta}$ is reflexive in $\underline{\operatorname{Eunc}}(\mathcal{I}, \mathcal{C})_{\mathrm{rel} \Theta}$ (i.e., the inclusion functor has a left adjoint). 
Proof. The reflector functor $\underline{\ell \mathrm{Func}}(\mathcal{I}, \mathcal{C})_{\text {rel } \Theta} \rightarrow \underline{\operatorname{Func}}(\mathcal{I}, \mathcal{C})_{\text {rel } \Theta}$ takes a lax functor $\mathbf{x}: \mathcal{I} \rightarrow \mathcal{C}$ to the unique functor $\widetilde{\mathbf{x}}$ that extends the morphism restriction of $\mathbf{x}$ to the graph. Similarly, a deformation $f$ : $\mathbf{x} \Rightarrow \mathbf{y}$ is taken to the unique deformation $\widetilde{f}: \widetilde{\mathbf{x}} \Rightarrow \widetilde{\mathbf{y}}$ that extends the deformation restriction of $f$ to the graph (according to (4)). The counit of the adjunction is the identity and the unit takes any lax functor $\mathbf{x}$ to the deformation $\varepsilon: \mathbf{x} \Rightarrow \widetilde{\mathbf{x}}$ which is inductively defined, using coherence (2), by the equalities:

- $\varepsilon_{i}=I d_{x_{i}}$, for any object $i \in \mathcal{I}$,

- $\varepsilon_{\tau}=I d_{x_{\tau}}$, for all $\tau \in \mathcal{G}$ and

- $\varepsilon_{\sigma \tau}=\varepsilon_{\sigma} x_{\tau} \circ x_{\sigma, \tau}$, for any composable pair of arrows $i \stackrel{\tau}{\longrightarrow} j \stackrel{\sigma}{\longrightarrow} k$ with $\tau \in \mathcal{G}$ and $\sigma \in \mathcal{I}$.

Simplicial SPACES AND THEIR REALIZATIONs. The simplicial category $\Delta$ has as objects the ordered sets $[n]=\{0,1, \ldots, n\}, n \geq 0$, and as arrows the (weakly) monotone maps $\alpha:[n] \rightarrow[m]$. This category is generated by the directed graph with edges all maps

$$
[n+1] \stackrel{\sigma_{i}}{\longrightarrow}[n] \stackrel{\delta_{i}}{\longleftarrow}[n-1], \quad 0 \leq i \leq n,
$$

where

$$
\sigma_{i}(j)=\left\{\begin{array}{ll}
j & j \leq i \\
j-1 & j>i
\end{array} \quad \text { and } \quad \delta_{i}(j)= \begin{cases}j & j<i \\
j+1 & j \geq i .\end{cases}\right.
$$

A simplicial object in a category $\mathcal{E}$ is just a functor $S: \Delta^{o p} \rightarrow \mathcal{E}$. Such a functor amounts to a collection $\left\{S_{n}=S[n], n \geq 0\right\}$ of objects in $\mathcal{E}$ together with morphisms $d_{i}=S\left(\delta_{i}\right): S_{n} \rightarrow S_{n-1}$ and $s_{i}=S\left(\sigma_{i}\right): S_{n} \rightarrow S_{n+1}, 0 \leq i \leq n$, called face and degeneracy operators respectively, which satisfy the simplicial identities described, for example, in [16, Definition 1.1]. A simplicial morphism $f: S \rightarrow S^{\prime}$ is just a natural transformation from $S$ to $S^{\prime}$, it then consists of a family $\left\{f_{n}=f_{[n]}: S_{n} \rightarrow S_{n+1}^{\prime}, n \geq 0\right\}$ of arrows in $\mathcal{E}$ which commute with the face and degeneracy operators. If $f, g: S \rightarrow S^{\prime}$ are simplicial morphisms, then a simplicial homotopy $H: f \Rightarrow g$ is a system $\left\{H_{m}: S_{n} \rightarrow S_{n+1}^{\prime}, 0 \leq m \leq n\right\}$ of arrows in $\mathcal{E}$ that satisfies the set of homotopy identities described, for example, in [16, Definition 5.1].

A simplicial space is a simplicial object in the category Top, of topological spaces, and a simplicial set is a simplicial object in the category Set, of sets. By regarding a set as a discrete space, any simplicial set is viewed as a simplicial space. Given a simplicial space $S$, Segal [21] defines its realization $|S|$ as follows: for each $n \geq 0$, let 
$\Delta^{n}$ denote the standard $n$-dimensional affine simplex and for each map $\alpha:[n] \rightarrow[m]$ in $\Delta$ let $\alpha_{*}: \Delta^{n} \rightarrow \Delta^{m}$ be the induced affine map (i.e., $\alpha_{*}\left(t_{0}, \ldots, t_{n}\right)=\left(t_{0}^{\prime}, \ldots, t_{m}^{\prime}\right)$ with $\left.t_{i}^{\prime}=\sum_{\alpha(i)=j} t_{j}\right)$. Then the space $|S|$ is defined from the topological sum $\coprod_{n>0} S_{n} \times \Delta^{n}$ by identifying $(S(\alpha)(x), t) \in S_{n} \times \Delta^{n}$ with $\left(x, \alpha_{*}(t)\right) \in S_{m} \times \Delta^{m}$, for all $x \in S_{m}, t \in \Delta^{n}$ and $\alpha:[n] \rightarrow[m]$ in $\Delta$. This construction is functorial and any simplicial homotopy $H: f \Rightarrow g$, between simplicial space morphisms $f, g: S \rightarrow S^{\prime}$, determines a homotopy $\widetilde{H}:|f| \Rightarrow|g|,[17$, Corollary 11.10]. A simplicial map that induces a homotopy equivalence on realizations is called a weak homotopy equivalence.

A bisimplicial set is a functor $S: \Delta^{o p} \times \Delta^{o p} \rightarrow$ Set. This amounts to a family of sets $\left\{S_{p, q}=S([p],[q]) ; 0 \leq p, q\right\}$ together with horizontal and vertical face and degeneracy operators

$$
S_{p+1, q} \stackrel{s_{i}^{h}}{\longleftarrow} S_{p, q} \stackrel{d_{i}^{h}}{\longrightarrow} S_{p-1, q} \text { and } S_{p, q+1} \stackrel{s_{j}^{v}}{\longleftarrow} S_{p, q} \stackrel{d_{j}^{v}}{\longrightarrow} S_{p, q-1},
$$

with $0 \leq i \leq p$ and $0 \leq j \leq q$ respectively, such that, for all $p$ and $q$, both $S_{p, *}$ and $S_{*, q}$ are simplicial sets and the horizontal operators commute with the vertical ones.

We now note that, on the one hand, any bisimplicial set $S$ provides two simplicial objects in the category of simplicial sets: the horizontal one $S^{h}:[p] \mapsto S_{p, *}$ and the vertical one $S^{v}:[q] \mapsto S_{*, q}$. Then, by taking realization, $S$ gives rise to two simplicial spaces $\left|S^{h}\right|:[p] \mapsto\left|S_{p, *}\right|$ and $\left|S^{v}\right|:[q] \mapsto\left|S_{*, q}\right|$, respectively. On the other hand, by composing with the diagonal functor diag : $\Delta^{o p} \rightarrow \Delta^{o p} \times \Delta^{o p}$, the bisimplicial set $S$ also provides another simplicial set $\operatorname{diag} S:[n] \mapsto S_{n, n}$, whose face and degeneracy operators are given in terms of those of $S$ by the formulas $d_{i}=d_{i}^{h} d_{i}^{v}$ and $s_{i}=s_{i}^{h} s_{i}^{v}$, respectively. It is known (e.g. [13, Lemma in page 86]) that there are natural homeomorphisms

$$
|| S^{h}\|\cong|\operatorname{diag} S| \cong\| S^{v} \| .
$$

We also point out (see $[3,1.2,4.3]$ for example) that if $f: S \rightarrow S^{\prime}$ is a bisimplicial morphism such that the induced maps $\left|f_{p, *}\right|:\left|S_{p, *}\right| \rightarrow\left|S_{p, *}^{\prime}\right|$ (resp. $\left|f_{*, q}\right|:\left|S_{*, q}\right| \rightarrow\left|S_{*, q}^{\prime}\right|$ ) are homotopy equivalences for all $p$ (resp. $q)$, then so is the map $|\operatorname{diag} f|:|\operatorname{diag} S| \rightarrow\left|\operatorname{diag} S^{\prime}\right|$.

Nerves of CATEgories AND THEIR ClASSifying SPACES. Any ordered set $[n]$ can be considered as a category with the elements of $[n]$ as objects and only one arrow $i \rightarrow j$ when $i \leq j$. After this identification, the category $\Delta$ can be considered as a full subcategory of the category Cat of small categories. The nerve NC, of a category $\mathcal{C}$, is the simplicial set defined as the restriction of the Yoneda 
embedding $\underline{\operatorname{Func}}(-, \mathcal{C}): \mathbf{C a t}^{o p} \rightarrow$ Set to the category $\Delta^{o p}$; thus $\mathrm{N}_{n} \mathcal{C}=\underline{\text { Func }}([n], \mathcal{C})$, for each $n \geq 0$.

Since the category $[n]$ is the free category generated by the graph $0 \rightarrow 1 \rightarrow \cdots n-1 \rightarrow n$, to give a functor $\mathbf{x}:[n] \rightarrow \mathcal{C}$, say $\mathbf{x}(i \rightarrow j)=$ $x_{i} \stackrel{x_{i, j}}{\longrightarrow} x_{j}$, is equivalent to giving a string of composable arrows in $\mathcal{C}$

$$
x_{0} \stackrel{x_{0,1}}{\longrightarrow} x_{1} \stackrel{x_{1,2}}{\longrightarrow} x_{2} \rightarrow \cdots x_{n-1} \stackrel{x_{n-1, n}}{\longrightarrow} x_{n} .
$$

Therefore we can make the identification

$$
\mathrm{N}_{n} \mathcal{C}=\coprod_{x_{0}, \ldots, x_{n} \in \operatorname{Ob}(\mathcal{C})} \mathcal{C}\left(x_{0}, x_{1}\right) \times \ldots \mathcal{C}\left(x_{n-1}, x_{n}\right)
$$

and thus $\mathrm{NC}$ can be described as the simplicial set whose set of $n$ simplices are strings of $n$ composable arrows in $\mathcal{C}$ and $\mathrm{N}_{0} \mathcal{C}=O b(\mathcal{C})$. The $i$-face (resp. degeneracy) operator of an $n$-simplex such as (6) in $\mathrm{NC}$ is obtained by deleting the object $x_{i}$, putting $x_{j}$ at place $j-1$ for all $j>i$ and using composition, if needed, to complete the new $(n-1)$-simplex (resp. replacing the object $x_{i}$ by the arrow identity $\left.I d_{x_{i}}: x_{i} \rightarrow x_{i}\right)$.

By applying realization to $\mathrm{NC}$, we obtain the classifying space $\mathrm{BC}$ of the category $\mathcal{C}$, that is $\mathrm{BC}=|\mathrm{NC}|$. This construction is functorial so that we have a functor B from Cat to the category of CW-complexes and cellular maps. We recall that functors related by natural transformations go to homotopic simplicial maps [11, VI, Proposition 2.2.2] and then to homotopic cellular maps. As a consequence, if a functor $F: \mathcal{C} \rightarrow \mathcal{C}^{\prime}$ has a left or right adjoint then the induced map $\mathrm{B} F: \mathrm{BC} \rightarrow \mathrm{BC}^{\prime}$ is a homotopy equivalence.

Nerves of 2-CATEGORIES AND Their ClASSIFYing SPACES. For any 2 -category $\mathcal{C}$, its nerve $\mathrm{NC}$ is the simplicial category (i.e., the simplicial object in Cat) obtained by restricting the functor $\operatorname{Func}(-, \mathcal{C})_{\operatorname{relOb}(-)}$ : Cat $^{o p} \rightarrow$ Cat to the category $\Delta^{o p}$; thus

$$
\mathrm{N}_{n} \mathcal{C}=\underline{\text { Func }}([n], \mathcal{C})_{\text {relOb }[n]},
$$

for each $n \geq 0$. Observe that the simplicial set of objects of $\mathrm{NC}$ coincides with the nerve of the underlying category of $\mathcal{C}$. Moreover, since $[n]$ is a free category, by the isomorphism (4), then giving an arrow $f: \mathbf{x} \rightarrow \mathbf{y}$ in $\mathrm{N}_{n} \mathcal{C}$ (i.e., a lax natural transformation relative to the objects of $[n]$ ) is equivalent to giving the string of deformations in $\mathcal{C}$,

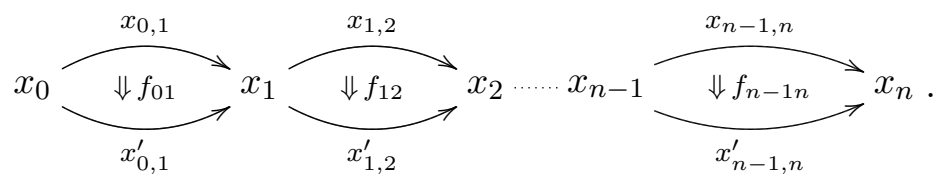


Therefore we can make the identification (cf. [12])

$$
\mathrm{N}_{n} \mathcal{C}=\coprod_{x_{0}, \ldots, x_{n} \in \mathcal{C}} \mathcal{C}\left(x_{0}, x_{1}\right) \times \mathcal{C}\left(x_{1}, x_{2}\right) \times \cdots \times \mathcal{C}\left(x_{n-1}, x_{n}\right)
$$

and $\mathrm{N}_{0} \mathcal{C}=O b(\mathcal{C})$, as a discrete category. After the above identification, the face and degeneracy functors are defined in the standard way.

The classifying space $\mathrm{BC}$ of the 2-category $\mathcal{C}$ is, by definition, the realization of the simplicial space obtained by composing $\mathrm{NC}: \Delta^{o p} \rightarrow$ Cat with the classifying space functor B : Cat $\rightarrow$ Top, that is,

$$
\mathrm{BC}=|\mathrm{BNC}|
$$

Note that, for each $n \geq 0, \mathrm{BN}_{n} \mathcal{C}=\left|\mathrm{NN}_{n} \mathcal{C}\right|$. Then, we have a natural homeomorphism

$$
\mathrm{BC} \cong|\operatorname{diagNNC}|,
$$

where $\mathrm{NNC}:([p],[q]) \mapsto \mathrm{N}_{p} \mathrm{~N}_{q} \mathcal{C}$ is the bisimplicial set double nerve of the 2 -category $\mathcal{C}$. This construction of classifying space defines a functor from the category of 2-categories to the category of $\mathrm{CW}$-complexes and cellular maps.

\section{Realizations of geometric nerves are classifying spaces}

It is not difficult to see that the data for an $n$-simplex $\mathbf{x}$ of the geometric nerve $\Delta \mathcal{C}$ of a 2-category $\mathcal{C}$, as described in the introduction, is the same as the data for a lax functor $\mathbf{x}:[n] \rightarrow \mathcal{C}$. That is, $\mathbf{x}$ consists of a family

$$
\mathbf{x}=\left\{x_{i}, x_{i, j}, x_{i, j, k}\right\}_{0 \leq i \leq j \leq k \leq n}
$$

with $x_{i}$ objects, $x_{i, j}: x_{i} \rightarrow x_{j}$ arrows and $x_{i, j, k}: x_{i, k} \Rightarrow x_{j, k} x_{i, j}$ deformations in $\mathcal{C}$, respectively, such that

- $x_{i, i}=I d_{x_{i}}, 0 \leq i \leq n$,

- $x_{i, j, j}=I d_{x_{i, j}}=x_{i, i, j}, 0 \leq i \leq j \leq n$, and

- $x_{k, \ell} x_{i, j, k} \circ x_{i, k, \ell}=x_{j, k, \ell} x_{i, j} \circ x_{i, j, \ell}, 0 \leq i \leq j \leq k \leq \ell \leq n$.

Thus, the simplicial set $\Delta \mathcal{C}$ can be described as the functor that takes each ordered set $[n]$ to the set of lax functors from $[n]$ to $\mathcal{C}$. The face operators $d_{m}=\delta_{m}^{*}: \Delta_{n} \mathcal{C} \rightarrow \Delta_{n-1} \mathcal{C}$ act by deleting all the elements indexed by $m$ and renumbering every index $\ell>m$ as $\ell-1$, that is, for any $\mathbf{x} \in \Delta_{n} \mathcal{C},\left(d_{m} \mathbf{x}\right)_{i}=x_{\delta_{m}(i)},\left(d_{m} \mathbf{x}\right)_{i, j}=x_{\delta_{m}(i), \delta_{m}(j)}$ and $\left(d_{m} \mathbf{x}\right)_{i, j, k}=$ $x_{\delta_{m}(i), \delta_{m}(j), \delta_{m}(k)}$. Analogously, the degeneracy operators are $s_{m}=\sigma_{m}^{*}$ : $\Delta_{n} \mathcal{C} \rightarrow \Delta_{n+1} \mathcal{C}$. 
As we said in the introduction, we devote this section to proving Theorem 1. To this end, we are going to build a diagram of simplicial sets and natural maps,

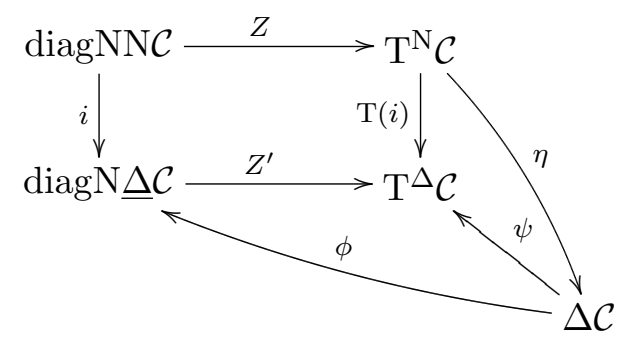

in which both the square and the triangle placed at the bottom of the diagram are commutative (i.e., $Z^{\prime} i=\mathrm{T}(i) Z$ and $\left.\psi=Z^{\prime} \phi\right)$ and the other triangle is commutative only up to homotopy (so that we will find a simplicial homotopy $H: \mathrm{T}(i) \Rightarrow \psi \eta$ ). Then we will observe that the maps $i, Z, Z^{\prime}$ and $\phi$ all induce homotopy equivalences on realizations and we conclude that so does every other map in the diagram. In particular, we will have that the composition $\eta Z$ induces a natural homotopy equivalence $\mathrm{BC} \stackrel{\sim}{\longrightarrow}|\Delta \mathcal{C}|$ and Theorem 1 will be proved.

The remainder of Section 1 is devoted to carrying out the above objectives, and so we naturally divide it into five subsections.

\subsection{Using the Artin-Mazur T-Construction: the simplicial SET $\mathrm{T}^{\mathrm{N}} \mathcal{C}$ AND THE MAPS $Z$ AND $\eta$}

There is another way of associating a simplicial set $\mathrm{T}^{\mathrm{N}} \mathcal{C}$ to a 2-category $\mathcal{C}$, whose simplices also have a pleasing geometric description. This simplicial nerve is defined by applying Artin-Mazur "Total simplicial set" construction [1, Section III] to the bisimplicial set NNC double nerve of $\mathcal{C}$ that we have already introduced.

Recall that, for a bisimplicial set $S$, the Artin-Mazur total simplicial set $\mathrm{T}(S)$ is defined as

$$
\mathrm{T}_{n}(S)=\left\{\left(x_{0}, x_{1}, \ldots, x_{n}\right) \in \prod_{i=0}^{n} S_{i, n-i} ; d_{0}^{v} x_{i}=d_{i+1}^{h} x_{i+1}, 0 \leq i<n\right\},
$$

$n \geq 0$, with face and degeneracy operators given by

$$
\begin{gathered}
\mathrm{T}_{n+1}(S) \stackrel{S_{i}}{\longleftarrow} \mathrm{T}_{n}(S) \stackrel{D_{i}}{\longrightarrow} \mathrm{T}_{n-1}(S), \\
D_{i}\left(x_{0}, \ldots, x_{n}\right)=\left(d_{i}^{v} x_{0}, d_{i-1}^{v} x_{1}, \ldots, d_{1}^{v} x_{i-1}, d_{i}^{h} x_{i+1}, d_{i}^{h} x_{i+2}, \ldots, d_{i}^{h} x_{n}\right), \\
S_{i}\left(x_{0}, \ldots, x_{n}\right)=\left(s_{i}^{v} x_{0}, s_{i-1}^{v} x_{1}, \ldots, s_{0}^{v} x_{i}, s_{i}^{h} x_{i}, s_{i}^{h} x_{i+1}, \ldots, s_{i}^{h} x_{n}\right) .
\end{gathered}
$$


Also recall that Zisman proves that there is a natural comparison map

$$
Z=Z_{S}: \operatorname{diag} S \rightarrow \mathrm{T}(S),
$$

which takes a $n$-simplex $x \in \operatorname{diag} S$ to

$$
Z(x)=\left(\left(d_{1}^{h}\right)^{n} x,\left(d_{2}^{h}\right)^{n-1} d_{0}^{v} x, \ldots,\left(d_{i+1}^{h}\right)^{n-i}\left(d_{0}^{v}\right)^{i} x, \ldots,\left(d_{0}^{v}\right)^{n} x\right) .
$$

This simplicial map induces a homotopy equivalence on realizations $|Z|:|\operatorname{diag} S| \stackrel{\sim}{\longrightarrow}|T(S)|$ (cf. [8]).

The simplicial set $\mathrm{T}^{\mathrm{N}} \mathcal{C}$ is defined as $\mathrm{T}^{\mathrm{N}} \mathcal{C}=\mathrm{T}(\mathrm{NNC})$. We then have the canonical map

$$
Z=Z_{\mathrm{NNC}}: \operatorname{diagNNC} \longrightarrow \mathrm{T}^{\mathrm{N}} \mathcal{C}
$$

which induces a homotopy equivalence

$$
|Z|: \mathrm{BC} \stackrel{\sim}{\longrightarrow}\left|\mathrm{T}^{\mathrm{N}} \mathcal{C}\right|,
$$

therefore the simplicial set $\mathrm{T}^{\mathrm{N}} \mathcal{C}$ also realizes the classifying space of the 2-category $\mathcal{C}$.

The simplices of $\mathrm{T}^{\mathrm{N}} \mathcal{C}$ have the following geometric description: the 0 - and 1-simplices of $\mathrm{T}^{\mathrm{N}} \mathcal{C}$ are the objects and arrows of $\mathcal{C}$ respectively, its 2 -simplices are diagrams in $\mathcal{C}$ of the form

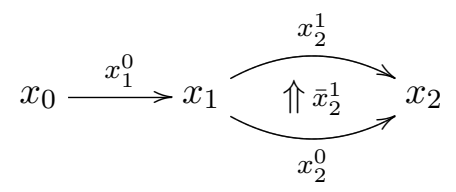

with $\bar{x}_{2}^{1}: x_{2}^{0} \Rightarrow x_{2}^{1}$ a deformation in $\mathcal{C}$, its 3 -simplices are diagrams in $\mathcal{C}$ of the form

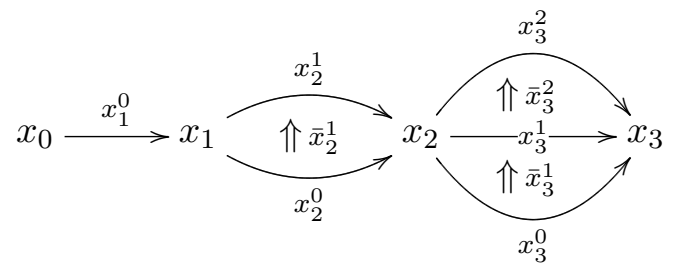

and so on. More precisely, an $n$-simplex $\mathbf{x}$ of $\mathrm{T}^{\mathrm{N}} \mathcal{C}$ is a family

$$
\mathbf{x}=\left\{x_{i}, x_{j}^{i}, \bar{x}_{j}^{i}\right\}
$$

consisting of objects $x_{i} \in \mathcal{C}$, for $0 \leq i \leq n$, arrows $x_{j}^{i}: x_{j-1} \rightarrow x_{j}$, for $0 \leq i<j \leq n$, and deformations $\bar{x}_{j}^{i}: x_{j}^{i-1} \Rightarrow x_{j}^{i}$, for $0<i<j \leq n$. 
The face operators $D_{m}: \mathrm{T}_{n}^{\mathrm{N}} \mathcal{C} \rightarrow \mathrm{T}_{n-1}^{\mathrm{N}} \mathcal{C}, 0 \leq m \leq n$, are given by the formulas

$$
\left(D_{m} \mathbf{x}\right)_{i}=x_{\delta_{m}(i)}, \quad\left(D_{m} \mathbf{x}\right)_{j}^{i}= \begin{cases}x_{\delta_{m}(j)}^{\delta_{m}(i)} & j \neq m \\ x_{m+1}^{i} x_{m}^{i} & j=m,\end{cases}
$$

and

$$
\left(\overline{D_{m} \mathbf{x}}\right)_{j}^{i}= \begin{cases}\bar{x}_{\delta_{m}(j)}^{\delta_{m}(i)} & i \neq m \neq j \\ \bar{x}_{m+1}^{i} \bar{x}_{m}^{i} & j=m \\ \bar{x}_{j+1}^{m+1} \circ \bar{x}_{j+1}^{m} & i=m,\end{cases}
$$

and the degeneracy operators $S_{m}: \mathrm{T}_{m}^{\mathrm{N}} \mathcal{C} \rightarrow \mathrm{T}_{m+1}^{\mathrm{N}} \mathcal{C}, 0 \leq m \leq n$, are given by

$$
\left(S_{m} \mathbf{x}\right)_{i}=x_{\sigma_{m}(i)}, \quad\left(S_{m} \mathbf{x}\right)_{j}^{i}=\left\{\begin{array}{cc}
x_{\sigma_{m}(j)}^{\sigma_{m}(i)} & j \neq m+1 \\
I d_{x_{m}} & j=m+1
\end{array}\right.
$$

and

$$
\left(\overline{S_{m} \mathbf{x}}\right)_{j}^{i}= \begin{cases}\bar{x}_{\sigma_{m}(j)}^{\sigma_{m}(i)} & i \neq m+1 \neq j \\ I d_{I d_{x_{m}}} & j=m+1 \\ I d_{x_{m+1}^{m}} & i=m+1 .\end{cases}
$$

We are now ready to define the simplicial map

$$
\eta: \mathrm{T}^{\mathrm{N}} \mathcal{C} \rightarrow \Delta \mathcal{C} .
$$

This map $\eta$ is the identity at levels zero and one, at level two it takes a 2 -simplex as (10) to the 2 -simplex of $\Delta \mathcal{C}$ represented by the triangle

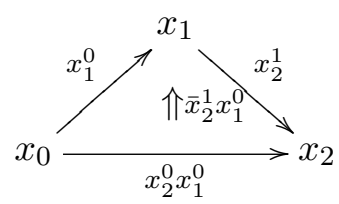

and, in general, $\eta$ takes an $n$-simplex $\mathbf{x} \in \mathrm{T}^{\mathrm{N}} \mathcal{C}$ as (11) to the geometric $n$-simplex $\eta \mathbf{x}$ given by

$$
\begin{aligned}
& (\eta \mathbf{x})_{i}=x_{i}, \\
& (\eta \mathbf{x})_{i, j}=x_{j}^{i} \ldots x_{i+1}^{i} \\
& (\eta \mathbf{x})_{i, j, k}=\left(\bar{x}_{k}^{j} \circ \ldots \circ \bar{x}_{k}^{i+1}\right) \ldots\left(\bar{x}_{j+1}^{j} \circ \ldots \circ \bar{x}_{j+1}^{i+1}\right) x_{j}^{i} \ldots x_{i+1}^{i}
\end{aligned}
$$

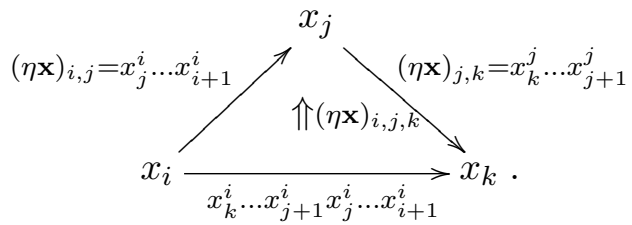




\subsection{The Simplicial CATEgory $\underline{\Delta C}$ And the map $i: \mathrm{NNC} \rightarrow \mathrm{N} \underline{\Delta} \mathcal{C}$}

The geometric nerve $\Delta \mathcal{C}$ of a category $\mathcal{C}$ is the simplicial set of objects of a simplicial category $\underline{\Delta C}$, which is defined by

$$
\underline{\Delta C}=\underline{\ell \text { Func }}(-, \mathcal{C})_{\text {relOb[-] }}: \Delta^{o p} \longrightarrow \text { Cat. }
$$

Thus, each category $\Delta_{n} \mathcal{C}$ has $\Delta_{n} \mathcal{C}$ as set of objects and its arrows are deformations relative to the set of objects of $[n]$. Therefore, in order to have an arrow $f: \mathbf{x} \rightarrow \mathbf{x}^{\prime}$ in $\underline{\Delta}_{n} \mathcal{C}$ we need $x_{i}=x_{i}^{\prime}$, for all $0 \leq i \leq n$, and in this case $f$ consists of a family $f=\left\{f_{i, j}: x_{i, j} \Rightarrow x_{i, j}^{\prime}\right\}_{0 \leq i \leq j \leq n}$ of deformations in $\mathcal{C}$, which satisfy the equations

- $f_{i, i}=I d_{x_{i}}, 0 \leq i \leq n$ and

- $f_{j, k} f_{i, j} \circ x_{i, j, k}=x_{i, j, k}^{\prime} \circ f_{i, k}, \quad 0 \leq i \leq j \leq k \leq n$.

The face and degeneracy functors are $d_{m}=\delta_{m}^{*}: \underline{\Delta}_{n} \mathcal{C} \rightarrow \underline{\Delta}_{n-1} \mathcal{C}$ and $s_{m}=\sigma_{m}^{*}: \underline{\Delta}_{n} \mathcal{C} \rightarrow \underline{\Delta}_{n+1} \mathcal{C}$ respectively, so that they act on objects as in the geometric nerve of $\mathcal{C}$, and for arrows $f: \mathbf{x} \rightarrow \mathbf{x}^{\prime}$ in $\underline{\Delta}_{n} \mathcal{C}$,

$$
\left(d_{m} f\right)_{i, j}=f_{\delta_{m}(i), \delta_{m}(j)} \quad \text { and } \quad\left(s_{m} f\right)_{i, j}=f_{\sigma_{m}(i), \sigma_{m}(j)} .
$$

This simplicial category $\underline{\Delta} \mathcal{C}$ defines, by taking the nerve of each category $\underline{\Delta}_{n} \mathcal{C}$, a bisimplicial set

$$
\mathrm{N} \triangleq \mathcal{C}:([p],[q]) \mapsto \mathrm{N}_{p} \Delta_{q} \mathcal{C} .
$$

More explicitly, an element $\chi \in \mathrm{N}_{p} \Delta_{q} \mathcal{C}$ can be described as a string

$$
\chi=\left(\mathbf{x}^{0} \stackrel{f^{1}}{\rightarrow} \mathbf{x}^{1} \rightarrow \cdots \rightarrow \mathbf{x}^{p-1} \stackrel{f^{p}}{\rightarrow} \mathbf{x}^{p}\right),
$$

of $p$ composable arrows in $\Delta_{q} \mathcal{C}$. The vertical face and degeneracy operators

$$
\mathrm{N}_{p} \underline{\Delta}_{q+1} \mathcal{C} \stackrel{s_{m}^{v}}{\longleftarrow} \mathrm{N}_{p} \underline{\Delta}_{q} \mathcal{C} \stackrel{d_{m}^{v}}{\longrightarrow} \mathrm{N}_{p} \underline{\Delta}_{q-1} \mathcal{C}, \quad 0 \leq m \leq q
$$

are induced by those of $\underline{\Delta C}$, that is

and

$$
d_{m}^{v}(\chi)=\left(d_{m} \mathbf{x}^{0} \stackrel{d_{m} f^{1}}{\longrightarrow} d_{m} \mathbf{x}^{1} \rightarrow \cdots \rightarrow d_{m} \mathbf{x}^{p-1} \stackrel{d_{m} f^{p}}{\longrightarrow} d_{m} \mathbf{x}^{p}\right)
$$

$$
s_{m}^{v}(\chi)=\left(s_{m} \mathbf{x}^{0} \stackrel{s_{m} f^{1}}{\longrightarrow} s_{m} \mathbf{x}^{1} \rightarrow \cdots \rightarrow s_{m} \mathbf{x}^{p-1} \stackrel{s_{m} f^{p}}{\longrightarrow} s_{m} \mathbf{x}^{p}\right) .
$$

The horizontal face and degeneracy operators

$$
\mathrm{N}_{p+1} \underline{\Delta}_{q} \mathcal{C} \stackrel{s_{m}^{h}}{\longleftarrow} \mathrm{N}_{p} \triangleq_{q} \mathcal{C} \stackrel{d_{m}^{h}}{\longrightarrow} \mathrm{N}_{p-1} \triangleq_{q} \mathcal{C}, 0 \leq m \leq q
$$


are those of the nerve $\mathrm{N} \underline{\Delta}_{q} \mathcal{C}$, that is, $d_{m}^{h}$ acts by deleting $\mathbf{x}^{m}$ in the string $\chi$, by putting $\mathbf{x}^{j}$ at place $j-1$ for all $j>m$ and by using composition, if needed, to form the new string $d_{m} \chi$. In the same way, $s_{m}^{h}$ duplicates $\mathbf{x}^{m}$ at place $m+1$ and introduces $I d_{\mathbf{x}^{m}}$ on the string.

We note now that the simplicial category $\mathrm{NC}$ is a simplicial subcategory of $\Delta \mathcal{C}$ via the inclusion functors

$$
i_{q}: \mathrm{N}_{q} \mathcal{C}=\underline{\operatorname{Func}}([q], \mathcal{C})_{\operatorname{rel} O b[q]} \hookrightarrow \underline{\ell \operatorname{Func}}([q], \mathcal{C})_{\operatorname{rel} O b[q]}=\underline{\Delta}_{q} \mathcal{C}, q \geq 0 .
$$

Then we have a bisimplicial inclusion

$$
i: \mathrm{NNC} \hookrightarrow \mathrm{N} \underline{\Delta C},
$$

whose restriction to the diagonal gives a simplicial inclusion, which we also denote as

$$
i: \operatorname{diagNNC} \rightarrow \operatorname{diagN} \underline{\Delta C} .
$$

Next, Theorem 2.1 proves that this inclusion is a weak homotopy equivalence.

THEOREM 2.1. For any 2-category $\mathcal{C}$, the map

$$
|i|: \mathrm{BC}=|\operatorname{diagNNC}| \stackrel{\sim}{\longrightarrow}|\operatorname{diagN} \underline{\mathcal{C}}|,
$$

induced by the inclusion (14) is a homotopy equivalence.

Proof. Since the category $[q]$ is free on a graph, by Proposition 1.1, each inclusion functor $i_{q}$ has a right adjoint. Therefore, the induced map on classifying spaces

$$
\mathrm{B} i_{q}: \mathrm{BN}_{q} \mathcal{C}=\left|\mathrm{NN}_{q} \mathcal{C}\right| \stackrel{\sim}{\longrightarrow} \mathrm{B} \Delta_{q} \mathcal{C}=\left|\mathrm{N} \Delta_{q} \mathcal{C}\right|
$$

is a homotopy equivalence, for all $q \geq 0$, whence Theorem 2.1 follows.

\subsection{Using the Artin-Mazur T Construction again: the} SIMPLICIAL SET $\mathrm{T}^{\Delta} \mathcal{C}$ AND THE MAPS $Z^{\prime}$ AND $\mathrm{T}(i)$

We now consider the simplicial category $\underline{\mathcal{C}}$, which was introduced in 2.2 , and then the total simplicial set

$$
\mathrm{T}^{\Delta} \mathcal{C}=\mathrm{TN} \underline{\mathcal{C}}
$$

associated to the bisimplicial set $\mathrm{N} \Delta \mathcal{C}$. At dimensions $\leq 1$, this simplicial set $\mathrm{T}^{\mathrm{N}} \mathcal{C}$ coincides with $\Delta \mathcal{C}$ (and also with $\mathrm{T}^{\mathrm{N}} \mathcal{C}$ ). Thus, the 0 - and 1-simplices of $\mathrm{T}^{\mathrm{N}} \mathcal{C}$ are the objects and arrows of $\mathcal{C}$, respectively. For $n \geq 2$, an $n$-simplex $\zeta$ of $\mathrm{T}^{\mathrm{N}} \mathcal{C}$ is a list

$$
\zeta=\left(\mathbf{x}^{n}, d_{0} \mathbf{x}^{n} \stackrel{f^{n-1}}{\longrightarrow} \mathbf{x}^{n-1}, \cdots, d_{0} \mathbf{x}^{2} \stackrel{f^{1}}{\longrightarrow} \mathbf{x}^{1}\right),
$$


in which each

$$
\mathbf{x}^{m}=\left(x_{i}^{m}, x_{i, j}^{m}: x_{i}^{m} \rightarrow x_{j}^{m}, x_{i, j, k}^{m}: x_{i, k}^{m} \Rightarrow x_{j, k}^{m} x_{i, j}^{m}\right) \in \Delta_{m} \mathcal{C}
$$

is a geometric $m$-simplex of $\mathcal{C}, 1 \leq m \leq n$, and each

$$
f^{m}=\left(f_{i, j}^{m}: x_{i+1, j+1}^{m+1} \Rightarrow x_{i, j}^{m}\right): d_{0} \mathbf{x}^{m+1} \rightarrow \mathbf{x}^{m}
$$

is a morphism in the category $\Delta_{m} \mathcal{C}\left(\right.$ so $x_{i}^{m}=x_{i+1}^{m+1}$ ), for all $1 \leq m<n$.

The face and degeneracy operators

$$
\mathrm{T}_{n+1}^{\mathrm{N}} \mathcal{C} \stackrel{S_{i}}{\longleftarrow} \mathrm{T}_{n}^{\mathrm{N}} \mathcal{C} \stackrel{D_{i}}{\longrightarrow} \mathrm{T}_{n-1}^{\mathrm{N}} \mathcal{C}, \quad 0 \leq m \leq n,
$$

on an $n$-simplex $\zeta \in \mathrm{T}^{\Delta} \mathcal{C}$ as (15) are given by the formulas:

$$
D_{m} \zeta= \begin{cases}\left(\mathbf{x}^{n-1}, f^{n-2}, \ldots, f^{1}\right) & m=0 \\ \left(d_{m} \mathbf{x}^{n}, d_{m-1} f^{n-1}, \ldots, d_{1} f^{n-m+1}, f^{n-m-1} d_{0} f^{n-m}, f^{n-m-2}, \ldots, f^{1}\right) & 0<m<n \\ \left(d_{n} \mathbf{x}^{n}, d_{n-1} f^{n-1}, \ldots, d_{2} f^{2}\right) & m=n,\end{cases}
$$

and

$$
S_{m} \zeta= \begin{cases}\left(s_{0} \mathbf{x}^{n}, \mathbf{x}^{n} \stackrel{I d}{\longrightarrow} \mathbf{x}^{n}, f^{n-1}, \ldots, f^{1}\right) & m=0 \\ \left(s_{m} \mathbf{x}^{n}, s_{m-1} f^{n-1}, \ldots, s_{0} f^{n-m}, \mathbf{x}^{n-m} \stackrel{I d}{\longrightarrow} \mathbf{x}^{n-m}, f^{n-m-1}, \ldots, f^{1}\right) & 0<m<n \\ \left(s_{n} \mathbf{x}^{n}, s_{n-1} f^{n-1}, \ldots, s_{1} f^{1}, d_{0} \mathbf{x}^{1} \stackrel{I d}{\longrightarrow} d_{0} \mathbf{x}^{1}\right) & m=n,\end{cases}
$$

for all $n \geq 2$.

Zisman's comparison map gives rise to the simplicial map

$$
Z^{\prime}=Z_{\mathrm{N} \Delta \mathcal{C}}: \operatorname{diagN} \underline{\Delta C} \rightarrow \mathrm{T}^{\Delta} \mathcal{C}
$$

which is a weak homotopy equivalence.

The simplicial map

$$
\mathrm{T}(i): \mathrm{T}^{\mathrm{N}} \mathcal{C} \rightarrow \mathrm{T}^{\Delta} \mathcal{C}
$$

is that induced by the bisimplicial inclusion (13) $i: \mathrm{NNC} \rightarrow \mathrm{N} \Delta \mathcal{C}$ on total complexes. It can be described as follows: Given an $n$-simplex $\mathbf{x}=$ $\left\{x_{i}, x_{j}^{i}, \bar{x}_{j}^{i}\right\} \in \mathrm{T}^{\mathrm{N}} \mathcal{C}$, let us write $\varsigma_{n} \mathbf{x} \in \Delta \mathcal{C}$ for the geometric $n$-simplex given by the family

$$
\left(\varsigma_{n} \mathbf{x}\right)_{i}=x_{i},\left(\varsigma_{n} \mathbf{x}\right)_{i, j}=x_{j}^{0} \cdots x_{i+1}^{0}, \text { and }\left(\varsigma_{n} \mathbf{x}\right)_{i, j, k}=I d_{\left(\varsigma_{n} \mathbf{x}\right)_{i, k}},
$$

and let $\widetilde{\varsigma}_{n-1} \mathbf{x}: d_{0} \varsigma_{n} \mathbf{x} \rightarrow \varsigma_{n-1} d_{0} \mathbf{x}$ be the arrow in $\triangleq_{n-1} \mathcal{C}$ given by the deformations

$$
\left(\widetilde{\varsigma}_{n-1} \mathbf{x}\right)_{i, j}=\bar{x}_{j+1}^{1} \cdots \bar{x}_{i+2}^{1}: x_{j+1}^{0} \cdots x_{i+2}^{0} \Rightarrow x_{j+1}^{1} \cdots x_{i+2}^{1} .
$$


Then, $\mathrm{T}(i)$ maps a simplex $\mathbf{x} \in \mathrm{T}_{n}^{\mathrm{N}} \mathcal{C}$ to

$$
\mathrm{T}(i) \mathbf{x}=\left(\varsigma_{n} \mathbf{x}, \widetilde{\varsigma}_{n-1} \mathbf{x}, \widetilde{\varsigma}_{n-2} d_{0} \mathbf{x}, \cdots, \widetilde{\varsigma}_{1} d_{0}^{n-2} \mathbf{x}\right) \in \mathrm{T}_{n}^{\Delta} \mathcal{C} .
$$

At this point we have completely described the commutative square

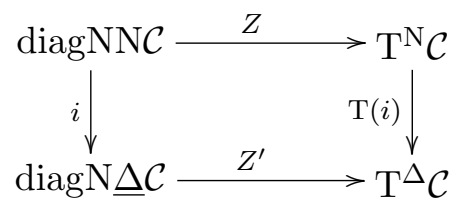

which forms part of the diagram (8) (the commutativity follows from the naturality of Zisman's maps). We also know that both $Z$ and $Z^{\prime}$ are weak homotopy equivalences and, by Theorem 2.1 , so is $i$. Therefore we conclude the following:

THEOREM 2.2. For any 2-category, the map

$$
|\mathrm{T}(i)|:\left|\mathrm{T}^{\mathrm{N}} \mathcal{C}\right| \stackrel{\sim}{\longrightarrow}\left|\mathrm{T}^{\Delta} \mathcal{C}\right|,
$$

induced by (17) on realizations, is a homotopy equivalence.

\subsection{ThE MAPS $\phi$ AND $\psi$}

As we showed in Subsection 2.2, the geometric nerve $\Delta \mathcal{C}$ of a 2-category $\mathcal{C}$ is the simplicial set of objects of the simplicial category $\Delta \mathcal{C}$. Hence, if we regard $\Delta \mathcal{C}$ as a discrete simplicial category (i.e., with only identities), we have an inclusion simplicial functor $\Delta \mathcal{C} \hookrightarrow \Delta \mathcal{C}$ that induces a bisimplicial map

$$
\phi: \Delta \mathcal{C} \hookrightarrow \mathrm{N} \underline{\mathcal{C}},
$$

where $\Delta \mathcal{C}$ is considered as a bisimplicial set that is constant in the horizontal direction (i.e., $(\underline{\Delta \mathcal{C}})_{p, q}=\mathrm{N}_{p} \Delta_{q} \mathcal{C}=\Delta_{q} \mathcal{C}$ ). Thus, for any $\mathbf{x} \in \Delta_{q} \mathcal{C}$,

$$
\phi_{p, q}(\mathbf{x})=\left(s_{0}^{h}\right)^{p}(\mathbf{x})=(\mathbf{x} \stackrel{I d}{\longrightarrow} \mathbf{x} \rightarrow \cdots \mathbf{x} \stackrel{I d}{\longrightarrow} \mathbf{x}) \in \mathrm{N}_{p} \Delta_{q} \mathcal{C} .
$$

We shall also write

$$
\phi: \Delta \mathcal{C} \hookrightarrow \operatorname{diagN} \underline{\underline{C}},
$$

for the induced simplicial map on diagonals.

LEMMA 2.3. For any 2-category $\mathcal{C}$ and any $p \geq 1$, the simplicial set $\mathrm{N}_{p-1} \underline{\underline{C}}$ is a simplicial deformation retract of $\mathrm{N}_{p} \underline{\Delta} \mathcal{C}$, via the simplicial injection $s_{0}^{h}: \mathrm{N}_{p-1} \underline{\Delta C} \hookrightarrow \mathrm{N}_{p} \underline{\mathcal{C}}$. 
Proof. Since $d_{0}^{h} s_{0}^{h}=I d$, to prove this lemma we only have to show the existence of a simplicial homotopy $H: i d \Rightarrow s_{0}^{h} d_{0}^{h}$.

In order to define $H$, we first associate a geometric $(q+1)$-simplex $\mu_{m} f \in \Delta \mathcal{C}$ to any arrow $f: \mathbf{x} \rightarrow \mathbf{y}$ in $\Delta_{q} \mathcal{C}$ and any $0 \leq m \leq q$. This simplex $\mu_{m} f$ is given by

$$
\left(\mu_{m} f\right)_{i}=x_{\sigma_{m}(i)}=y_{\sigma_{m}(i)}, \quad\left(\mu_{m} f\right)_{i, j}= \begin{cases}y_{i, j} & j \leq m \\ x_{\sigma_{m}(i), j-1} & m<j\end{cases}
$$

and

$$
\left(\mu_{m} f\right)_{i, j, k}= \begin{cases}y_{i, j, k} & k \leq m \\ x_{j, k-1} f_{i, j} \circ x_{i, j, k-1} & j \leq m<k \\ x_{\sigma_{m}(i), j-1, k-1} & m<j\end{cases}
$$

Observe that $\mu_{0} f=s_{0} \mathbf{y}$ and $d_{q+1} \mu_{q} f=\mathbf{y}$, for all $f: \mathbf{x} \rightarrow \mathbf{y}$, and that for $f=I d_{\mathbf{x}}$, any identity in $\Delta_{q} \mathcal{C}$, we have $\mu\left(I d_{\mathbf{x}}\right)=s_{m} \mathbf{x}$, for all $0 \leq m \leq q$. Next, we consider the arrows

$$
h_{m} f: s_{m} \mathbf{x} \rightarrow \mu_{m} f,
$$

in $\underline{\Delta}_{q+1} \mathcal{C}$, defined by

$$
\left(h_{m} f\right)_{i, j}=\left\{\begin{array}{ll}
I d_{y_{i, j}} & j \leq m \\
f_{\sigma_{m}(i), j-1} & m<j
\end{array},\right.
$$

(note that $h_{0}=s_{0}^{v}: \mathrm{N}_{1} \Delta_{q} \mathcal{C} \rightarrow \mathrm{N}_{1} \Delta_{q+1} \mathcal{C}$, and that $d_{q+1}^{v} h_{q} f=I d_{\mathbf{y}}$ for all $f: \mathbf{x} \rightarrow \mathbf{y}$ ). And finally we define the homotopy $H$ by the maps

$$
H_{m}: \mathrm{N}_{p} \Delta_{q} \mathcal{C} \rightarrow \mathrm{N}_{p} \Delta_{q+1} \mathcal{C}, \quad 0 \leq m \leq q
$$

which send a $q$-simplex $\chi=\left(\mathbf{x}^{0} \stackrel{f^{0}}{\rightarrow} \mathbf{x}^{1} \rightarrow \cdots \rightarrow \mathbf{x}^{p-1} \stackrel{f^{p}}{\rightarrow} \mathbf{x}^{p}\right)$ in $\mathrm{N} \underline{\mathcal{C}}$ to the $(q+1)$-simplex

$$
H_{m} \chi=\left(\mu_{m} f^{1} \stackrel{h_{m} f^{1}}{\longrightarrow} s_{m} \mathbf{x}^{1} \stackrel{s_{m} f^{2}}{\longrightarrow} \cdots \longrightarrow s_{m} \mathbf{x}^{p-1} \stackrel{s_{m} f^{p}}{\longrightarrow} s_{m} \mathbf{x}^{p}\right) .
$$

We leave it to the reader to check that $H$ is indeed a simplicial homotopy as required.

By an iterative application of above Lemma 2.3, the simplicial map

$$
\phi_{p, *}: \Delta \mathcal{C}=\mathrm{N}_{0} \underline{\Delta C} \rightarrow \mathrm{N}_{p} \underline{\Delta C}
$$

induces, for all $p \geq 0$, a homotopy equivalence on geometric realizations, and therefore so does the simplicial map $\phi: \Delta \mathcal{C} \rightarrow \operatorname{diagN} \underline{\Delta C}$. We have then proved Theorem 2.4 stated below. 
THEOREM 2.4. For any 2-category $\mathcal{C}$, the map

$$
|\phi|:|\Delta \mathcal{C}| \stackrel{\sim}{\longrightarrow}|\operatorname{diagN} \underline{\underline{C}}|
$$

induced by the above simplicial map (19) is a homotopy equivalence.

To fully complete the data in diagram (8) it only remains to introduce the simplicial map

$$
\psi: \Delta \mathcal{C} \rightarrow \mathrm{T}^{\Delta} \mathcal{C}
$$

which is defined to be the composite map $\Delta \mathcal{C} \stackrel{\phi}{\longrightarrow} \operatorname{diagN} \underline{\Delta C} \stackrel{Z^{\prime}}{\longrightarrow} \mathrm{T}^{\Delta} \mathcal{C}$, of the above $\phi(19)$ with Zisman map $Z^{\prime}$ (16). Explicitly, $\psi$ maps a simplex $\mathbf{x} \in \Delta_{n} \mathcal{C}$ to

$$
\psi(\mathbf{x})=\left(\mathbf{x}, d_{0} \mathbf{x} \stackrel{I d}{\longrightarrow} d_{0} \mathbf{x}, \cdots, d_{0}^{n-1} \mathbf{x} \stackrel{I d}{\longrightarrow} d_{0}^{n-1} \mathbf{x}\right) .
$$

Since $\phi$ and $Z^{\prime}$ are both weak homotopy equivalences, we have

THEOREM 2.5. For any 2-category $\mathcal{C}$, the map

$$
|\psi|:|\Delta \mathcal{C}| \stackrel{\sim}{\longrightarrow}\left|\mathrm{T}^{\Delta} \mathcal{C}\right|
$$

induced by the above simplicial map (20) is a homotopy equivalence.

\subsection{The MAP $|\eta|$ IS A HOMOTOPY EQUIVALENCE}

We are now going to prove that the triangle

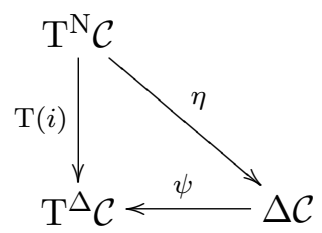

is commutative up to a simplicial homotopy. Then, since $\mathrm{T}(i)$ and $\psi$ are both weak homotopy equivalences, by Theorems 2.2 and 2.5, we will have proved that $|\eta|$ is also a homotopy equivalence.

PROPOSITION 2.6. There is a simplicial homotopy $H: \mathrm{T}(i) \Rightarrow \psi \eta$.

Proof. In order to define $H$, we first build a geometric $(n+1)$-simplex $h_{m} \mathbf{x} \in \Delta \mathcal{C}$, associated to any $n$-simplex $\mathbf{x} \in \mathrm{T}^{\mathrm{N}} \mathcal{C}$ and any $0 \leq m \leq n$. 
This simplex $h_{m} \mathbf{x}$ is given by

$$
\begin{aligned}
& \left(h_{m} \mathbf{x}\right)_{i}=x_{\sigma_{m}(i)}, \\
& \left(h_{m} \mathbf{x}\right)_{i, j}= \begin{cases}(\eta \mathbf{x})_{i, j}=x_{j}^{i} \cdots x_{i+1}^{i} & j \leq m \\
\left(\varsigma_{n} \mathbf{x}\right)_{\sigma_{m}(i), j-1}=x_{j-1}^{0} \cdots x_{\sigma_{m}(i)+1}^{0} & m<j\end{cases}
\end{aligned}
$$

and

$$
\begin{aligned}
& \left(h_{m} \mathbf{x}\right)_{i, j, k}= \\
& \left\{\begin{array}{lr}
(\eta \mathbf{x})_{i, j, k}=\left(\bar{x}_{k}^{j} \circ \cdots \circ \bar{x}_{k}^{i+1}\right) \cdots\left(\bar{x}_{j+1}^{j} \circ \cdots \circ \bar{x}_{j+1}^{i+1}\right) x_{j}^{i} \cdots x_{i+1}^{i} & k \leq m \\
x_{k}^{0} \cdots x_{j+1}^{0}\left(\bar{x}_{j}^{i} \circ \cdots \circ \bar{x}_{j}^{1}\right) \cdots\left(\bar{x}_{i+1}^{i} \circ \cdots \circ \bar{x}_{i+1}^{1}\right) & j \leq m<k \\
I d_{x_{k-1}^{0} \cdots x_{\sigma_{m}(i)+1}^{0}} & m<j .
\end{array}\right.
\end{aligned}
$$

Observe that $h_{0} \mathbf{x}=s_{0} \varsigma_{n} \mathbf{x}, h_{1} \mathbf{x}=s_{1} \varsigma_{n} \mathbf{x}$ and $d_{n+1} h_{n} \mathbf{x}=\eta \mathbf{x}$. Next, we consider the arrows

$$
\widetilde{h}_{m} \mathbf{x}: d_{0} h_{m} \mathbf{x} \rightarrow h_{m-1} d_{0} \mathbf{x}
$$

in $\underline{\Delta}_{n} \mathcal{C}$, for $m \geq 1$, defined by the family of deformations

$$
\left(\widetilde{h}_{m} \mathbf{x}\right)_{i, j}= \begin{cases}I d_{\left(\eta d_{0} \mathbf{x}\right)_{i, j}} & j<m \\ \bar{x}_{j}^{1} \cdots \bar{x}_{\sigma_{m}(i+1)+1}^{1} & m \leq j .\end{cases}
$$

And, finally, the homotopy $H$ is given by the maps

$$
H_{m}: \mathrm{T}_{n}^{\mathrm{N}} \mathcal{C} \rightarrow \mathrm{T}_{n+1}^{\Delta} \mathcal{C}, \quad 0 \leq m \leq n,
$$

which send a $n$-simplex $\mathbf{x}=\left\{x_{i}, x_{j}^{i}, \bar{x}_{j}^{i}\right\}$, in $\mathrm{T}^{\mathrm{N}} \mathcal{C}$ to the $(n+1)$-simplex

$$
\begin{aligned}
& H_{m} \mathbf{x}= \\
& \begin{cases}\left(h_{0} \mathbf{x}, I d_{\varsigma_{n} \mathbf{x}}, \widetilde{\varsigma}_{n-1} \mathbf{x}, \widetilde{\varsigma}_{n-2} d_{0} \mathbf{x}, \cdots, \widetilde{\varsigma}_{1} d_{0}^{n-2} \mathbf{x}\right) & m=0 \\
\left(h_{m} \mathbf{x}, \widetilde{h}_{m} \mathbf{x}, \widetilde{h}_{m-1} d_{0} \mathbf{x}, \cdots, \widetilde{h}_{1} d_{0}^{m-1} \mathbf{x}, I d_{\varsigma_{n-m}} d_{0}^{m} \mathbf{x}, \widetilde{\varsigma}_{n-m-1} d_{0}^{m} \mathbf{x}, \cdots, \widetilde{\varsigma}_{1} d_{0}^{n-2} \mathbf{x}\right) & 0<m<n \\
\left(h_{n} \mathbf{x}, \widetilde{h}_{n} \mathbf{x}, \widetilde{h}_{n-1} d_{0} \mathbf{x}, \cdots, \widetilde{h}_{1} d_{0}^{n-1} \mathbf{x}\right) & m=n\end{cases}
\end{aligned}
$$

(note that $H_{0}=s_{0} \mathrm{~T}(i)$ and $H_{1}=s_{1} \mathrm{~T}(i)$ ). To prove in full that $H$ is a simplicial homotopy between $\mathrm{T}(i)$ and $\psi \eta$, some verifications are needed, which are straightforward (though tedious) and we will therefore leave them to the reader.

As an immediate consequence of the comments at the beginning of this Section 2 and the above Proposition 2.6, we obtain Theorem 2.7 below, from which the proof of Theorem 1 in the introduction is completed. 
THEOREM 2.7. For any 2-category $\mathcal{C}$, the map

$$
|\eta|:\left|\mathrm{T}^{\mathrm{N}} \mathcal{C}\right| \stackrel{\sim}{\longrightarrow}|\Delta \mathcal{C}|
$$

induced by the simplicial map (12) is a homotopy equivalence.

Remark. A strict monoidal category $\mathcal{C}=(\mathcal{C}, \otimes, I)[15]$ can be viewed as a 2-category with only one object, say $I$, the objects $x$ of $\mathcal{C}$ as arrows $x: I \rightarrow I$ and the arrows of $\mathcal{C}$ as deformations. It is the horizontal composition of arrows and deformations given by the strict tensor $\otimes: \mathcal{C} \times \mathcal{C} \rightarrow \mathcal{C}$ and the vertical composition of deformations given by the composition of arrows in $\mathcal{C}$. Then, the classifying space $\mathrm{BC}$ of the monoidal category is just the classifying space of the 2-category it defines. Then, by Theorem 1 , the geometric nerve $\Delta \mathcal{C}$ realizes the classifying space of the monoidal category $\mathcal{C}$, that is, $\mathrm{BC} \simeq|\Delta \mathcal{C}|$.

The geometric nerve $\Delta \mathcal{C}$ is a 3 -coskeletal reduced simplicial set whose simplices have the following simplified interpretation: the 1simplices are the objects of $\mathcal{C}$, the 2-simplices are morphisms of $\mathcal{C}$ of the form

$$
x_{0,2} \stackrel{x_{0,1,2}}{\longrightarrow} x_{1,2} \otimes x_{0,1}
$$

and the 3 -simplices are commutative squares in $\mathcal{C}$ of the form

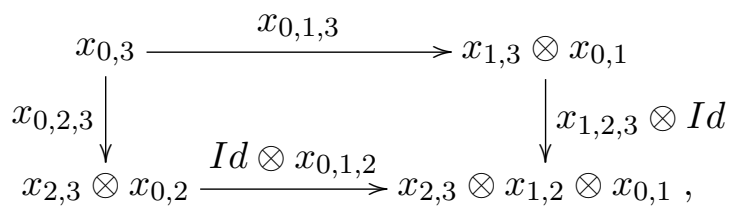

the face operator $d_{i}$ is obtained by deleting the data in which the index $i$ appears.

When the strict monoidal category $\mathcal{C}$ is a categorical group, that is, when all objects and arrows are invertible, it is largely known that $\mathrm{BC} \simeq|\Delta \mathcal{C}|$. Indeed, in that case $\mathcal{C}$ is just an internal category in the category of groups and the nerve $\mathrm{NC}$ is a simplicial group. Thus, we can apply Kan's classifying complex functor $\bar{W}[16]$ to $\mathrm{NC}$ and the resulting simplicial set is isomorphic to the geometric nerve of $\mathcal{C}$, that is, $\bar{W} \mathrm{NC} \cong \Delta \mathcal{C}$. We also recall that strict categorical groups are equivalent to crossed modules (see [15] for example) and that classifying spaces of crossed modules are defined as the classifying spaces of the categorical groups they define $([4,14])$. We finally note that geometric nerves of non-necessarily strict categorical groups have been treated in $[6,7]$ and, more generally, geometric nerves of arbitrary monoidal categories (even of bicategories) have been treated in [5] 


\section{A sufficient condition for a 2-functor to induce a homotopy equivalence}

In this section we prove Theorem 2, stated in the Introduction. Let us recall that Quillen's Theorem A [13] asserts that a functor $F: \mathcal{C} \rightarrow \mathcal{C}^{\prime}$ induces a homotopy equivalence $\mathrm{B} F: \mathrm{BC} \rightarrow \mathrm{BC}^{\prime}$ whenever the classifying space of every homotopy fiber category $y^{\prime} / F$ is contractible, for all objects $y^{\prime} \in \mathcal{C}^{\prime}$. The homotopy fiber category $y^{\prime} / F$ has as objects the pairs $\left(u^{\prime}, x\right)$, with $x \in \mathcal{C}$ an object and $u^{\prime}: y^{\prime} \rightarrow F(x)$ an arrow in $\mathcal{C}^{\prime}$. The arrows $u:\left(u_{0}^{\prime}, x_{0}\right) \rightarrow\left(u_{1}^{\prime}, x_{1}\right)$ in $y^{\prime} / F$ are arrows $u: x_{0} \rightarrow x_{1}$ in $\mathcal{C}$ such that $u_{1}^{\prime}=F(u) u_{0}^{\prime}$.

In section 1 we have recalled the notion of homotopy fiber 2-category $y^{\prime} / / F$, for any 2-functor $F: \mathcal{C} \rightarrow \mathcal{C}^{\prime}$ between 2-categories and any object $y^{\prime} \in \mathcal{C}^{\prime}$. In particular, by taking $F=I d_{\mathcal{C}}$, we have the lax comma 2categories $y / / \mathcal{C}=y / / I d_{\mathcal{C}}, y \in O b \mathcal{C}$. The objects of the 2-category $y / / \mathcal{C}$ are pairs $(v, x)$ with $v: y \rightarrow x$ an arrow, its arrows are also pairs $(f, u):(v, x) \rightarrow\left(v^{\prime}, x^{\prime}\right)$ with $u: x \rightarrow x^{\prime}$ an arrow and $f: v^{\prime} \Rightarrow u v$ a deformation, and its deformations $g:(f, u) \Rightarrow\left(f^{\prime}, u^{\prime}\right)$ are deformations $g: u \Rightarrow u^{\prime}$ such that $f^{\prime}=g v \circ f$.

As for the case of categories, we have:

PROPOSITION 3.1. Given a 2-category $\mathcal{C}$, for any object $y \in \mathcal{C}$, the classifying space $\mathrm{B}(y / / \mathcal{C})$ is contractible.

Proof. By Theorem 1 we have $\mathrm{B}(y / / \mathcal{C}) \simeq|\Delta(y / / \mathcal{C})|$. Let $\operatorname{Dec}(\Delta \mathcal{C})$ be the simplicial set obtained from the geometric nerve of $\mathcal{C}$ by "forgetting" the first face and degeneracy operators at each level and "renumbering" the levels [11, Chap. VI,1]. This is an augmented simplicial set over $\Delta_{0} \mathcal{C}=O b \mathcal{C}$, with augmentation given by the face operator $d_{1}: \Delta_{1} \mathcal{C} \rightarrow$ $\Delta_{0} \mathcal{C}$, on which the first degeneracy operators $s_{0}$ define a simplicial contraction.

Furthermore, the augmentation $d_{1}: \Delta_{1} \mathcal{C} \rightarrow \Delta_{0} \mathcal{C}$ gives rise to a decomposition of the simplicial set $\operatorname{Dec}(\Delta \mathcal{C})$ as

$$
\operatorname{Dec}(\Delta \mathcal{C})=\coprod_{y \in O b(\mathcal{C})} d_{1}^{-1}(y)
$$

in such a way that the above simplicial contraction of $\operatorname{Dec}(\Delta \mathcal{C})$ restricts to the simplicial subsets fibers $d_{1}^{-1}(y)$, for any object $y \in \mathcal{C}$. Therefore, all the simplicial sets $d_{1}^{-1}(y)$ are homotopically trivial. However, it is not difficult to identify each fiber $d_{1}^{-1}(y)$ with the geometric nerve $\Delta(y / / \mathcal{C})$ of the lax comma 2-category $y / / \mathcal{C}$ and so $|\Delta(y / / \mathcal{C})|$ is contractible.

We now are ready to prove our Theorem 2, an extension of Quillen's Theorem A for 2-categories. 


\subsection{Proof of Theorem 2}

Let $F: \mathcal{C} \rightarrow \mathcal{C}^{\prime}$ be a 2-functor such that, for any object $y^{\prime} \in \mathcal{C}^{\prime}$, the space $\mathrm{B}\left(y^{\prime} / / F\right)$ is contractible. We have to prove that the induced map $\mathrm{B} F: \mathrm{BC} \rightarrow \mathrm{BC}^{\prime}$ is a homotopy equivalence.

Hereafter, we shall also write $F: \Delta \mathcal{C} \rightarrow \Delta \mathcal{C}^{\prime}$ for the simplicial map induced by the 2-functor $F: \mathcal{C} \rightarrow \mathcal{C}^{\prime}$ between the corresponding geometric nerves.

Let us consider the bisimplicial set $S=S(F)$, which is given by:

$$
S_{p, q}=\left\{\left(\mathbf{x}, \mathbf{y}^{\prime}\right) \in \Delta_{p} \mathcal{C} \times \Delta_{p+q+1} \mathcal{C}^{\prime} ; F(\mathbf{x})=d_{0}^{q+1}\left(\mathbf{y}^{\prime}\right)\right\}, \quad p, q \geq 0,
$$

with horizontal and vertical face and degeneracy operators

$$
S_{p+1, q}<\stackrel{s_{i}^{h}}{<} S_{p, q} \stackrel{d_{i}^{h}}{\longrightarrow} S_{p-1, q} \text { and } S_{p, q+1} \ll \stackrel{s_{j}^{v}}{\longleftarrow} S_{p, q} \stackrel{d_{j}^{v}}{\longrightarrow} S_{p, q-1},
$$

given by

$$
\begin{aligned}
& d_{i}^{h}\left(\mathbf{x}, \mathbf{y}^{\prime}\right)=\left(d_{i} \mathbf{x}, d_{q+i+1} \mathbf{y}^{\prime}\right), d_{j}^{v}\left(\mathbf{x}, \mathbf{y}^{\prime}\right)=\left(\mathbf{x}, d_{j} \mathbf{y}^{\prime}\right), \\
& s_{i}^{h}\left(\mathbf{x}, \mathbf{y}^{\prime}\right)=\left(s_{i} \mathbf{x}, s_{q+i+1} \mathbf{y}^{\prime}\right), \quad s_{j}^{v}\left(\mathbf{x}, \mathbf{y}^{\prime}\right)=\left(\mathbf{x}, s_{j} \mathbf{y}^{\prime}\right) .
\end{aligned}
$$

We first observe that, for any $p$, the canonical projection

$$
\varphi_{p}: S_{p, 0} \rightarrow \Delta_{p} \mathcal{C}, \quad\left(\mathbf{x}, y^{\prime}\right) \mapsto \mathbf{x}
$$

defines an augmentation on the simplicial set $S_{p, *}$ over the set $\Delta_{p} \mathcal{C}$ and that these augmentations $\varphi_{p}$, since they commute with the horizontal face and degeneracy operators, define a bisimplicial map

$$
\varphi: S \rightarrow \Delta \mathcal{C}, \quad\left(\mathbf{x}, \mathbf{y}^{\prime}\right) \mapsto \mathbf{x},
$$

where $\Delta \mathcal{C}$ is considered as a constant bisimplicial set in the vertical direction.

Furthermore, every augmented simplicial set $S_{p, *} \rightarrow \Delta_{p} \mathcal{C}$ has a simplicial contraction, which is given by the maps $\varepsilon_{q+1}: S_{p, q} \rightarrow S_{p, q+1}, q \geq$ -1 , defined by

$$
\begin{aligned}
& \varepsilon_{0}(\mathbf{x})=\left(\mathbf{x}, s_{0} F(\mathbf{x})\right), \quad \mathbf{x} \in \Delta_{p} \mathcal{C} \\
& \varepsilon_{q+1}\left(\mathbf{x}, \mathbf{y}^{\prime}\right)=\left(\mathbf{x}, s_{q+1} \mathbf{y}^{\prime}\right), \quad\left(\mathbf{x}, \mathbf{y}^{\prime}\right) \in S_{p, q}, q \geq 0 .
\end{aligned}
$$

It follows that each induced map $\left|\varphi_{p}\right|:\left|S_{p, *}\right| \stackrel{\sim}{\rightarrow} \Delta_{p} \mathcal{C}$ is a homotopy equivalence and therefore the induced $\operatorname{map}|\operatorname{diag} \varphi|:|\operatorname{diag} S| \stackrel{\sim}{\rightarrow}|\Delta \mathcal{C}|$ is also a homotopy equivalence.

Secondly, we note that, for any $q$, the map

$$
\varphi_{q}^{\prime}: S_{0, q} \rightarrow \Delta_{q} \mathcal{C}^{\prime}, \quad\left(x, \mathbf{y}^{\prime}\right) \mapsto d_{q+1} \mathbf{y}^{\prime},
$$


gives an augmentation on $S_{*, q}$ over the set $\Delta_{q} \mathcal{C}^{\prime}$ and that these maps define a bisimplicial map

$$
\varphi^{\prime}: S \rightarrow \Delta \mathcal{C}^{\prime}, \quad\left(\mathbf{x}, \mathbf{y}^{\prime}\right) \mapsto d_{q+1} \mathbf{y}^{\prime},
$$

where $\Delta \mathcal{C}^{\prime}$ is considered as a bisimplicial set constant in the horizontal direction.

Let us now assume that we have proved that the induced map

$$
\left|\operatorname{diag} \varphi^{\prime}\right|:|\operatorname{diag} S| \rightarrow\left|\Delta \mathcal{C}^{\prime}\right|
$$

is a homotopy equivalence. This will be proved later, after Lemma 3.2 below.

Then, finally, we consider the following commutative diagram

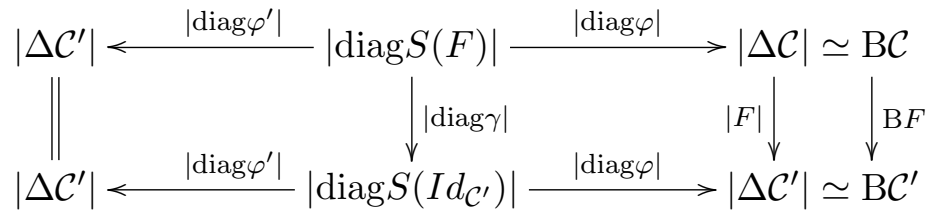

where $\gamma: S(F) \rightarrow S\left(I d_{\mathcal{C}^{\prime}}\right)$ is the bisimplicial map given by $\gamma_{p, q}\left(\mathbf{x}, \mathbf{y}^{\prime}\right)=$ $\left(F(\mathbf{x}), \mathbf{y}^{\prime}\right)$. In this diagram, all the horizontal maps are homotopy equivalences (note that, by Proposition 3.1 above, the homotopy fiber 2categories $y^{\prime} / / I d_{\mathcal{C}^{\prime}}=y^{\prime} / / \mathcal{C}^{\prime}$ are contractible). Therefore, first $|\operatorname{diag} \gamma|$ and then $|F|$ and $\mathrm{B} F$ are homotopy equivalences, and the proof will be complete.

It remains to prove that the induced map $\left|\operatorname{diag} \varphi^{\prime}\right|$ in (21) is a homotopy equivalence. To do so, we return to the proof of Theorem 2 .

For any $q \geq 0$, the augmentation $\varphi_{q}^{\prime}: S_{0, q} \rightarrow \Delta_{q}\left(\mathcal{C}^{\prime}\right)$ produces a decomposition of the simplicial set $S_{*, q}$ as

$$
S_{*, q}=\coprod_{\mathbf{y}^{\prime} \in \Delta_{q} \mathcal{C}^{\prime}} S_{*, q}\left(\mathbf{y}^{\prime}\right)
$$

where $S_{*, q}\left(\mathbf{y}^{\prime}\right)=\varphi_{q}^{\prime-1}\left(\mathbf{y}^{\prime}\right)$ is the fiber of $\varphi_{q}^{\prime}$ at $\mathbf{y}^{\prime}$. That is, $S_{*, q}\left(\mathbf{y}^{\prime}\right)$ is the simplicial subset of $S_{*, q}$ with

$$
S_{p, q}\left(\mathbf{y}^{\prime}\right)=\left\{\left(\mathbf{x}, \mathbf{z}^{\prime}\right) \in \Delta_{p} \mathcal{C} \times \Delta_{p+q+1} \mathcal{C}^{\prime} ; d_{0}^{q+1} \mathbf{z}^{\prime}=F(\mathbf{x}), d_{q+1}^{p+1} \mathbf{z}^{\prime}=\mathbf{y}^{\prime}\right\} .
$$

Observe that, for any $q \geq 1$ and any $\mathbf{y}^{\prime} \in \Delta_{q} \mathcal{C}^{\prime}$, the simplicial map $d_{q-1}^{v}: S_{*, q} \rightarrow S_{*, q-1}$ restricts to a simplicial map

$$
d_{q-1}^{v}: S_{*, q}\left(\mathbf{y}^{\prime}\right) \rightarrow S_{*, q-1}\left(d_{q-1} \mathbf{y}^{\prime}\right)
$$

and that we have: 
LEMMA 3.2. The induced map

$$
\left|d_{q-1}^{v}\right|:\left|S_{*, q}\left(\mathbf{y}^{\prime}\right)\right| \longrightarrow\left|S_{*, q-1}\left(d_{q-1} \mathbf{y}^{\prime}\right)\right|
$$

is a homotopy equivalence, for all $q \geq 1$ and all $\mathbf{y}^{\prime} \in \Delta_{q} \mathcal{C}^{\prime}$.

Proof. In fact, we are going to prove that $S_{*, q-1}\left(d_{q-1} \mathbf{y}^{\prime}\right)$ is a simplicial deformation retract of $S_{*, q}\left(\mathbf{y}^{\prime}\right)$, with $d_{q-1}^{v}$ as retraction and section given by the simplicial map

$$
\Gamma: S_{*, q-1}\left(d_{q-1} \mathbf{y}^{\prime}\right) \longrightarrow S_{*, q}\left(\mathbf{y}^{\prime}\right) ; \quad\left(\mathbf{x}, \mathbf{z}^{\prime}\right) \mapsto\left(\mathbf{x}, \mathbf{z}^{\prime \prime}\right)
$$

where, if $\left(\mathbf{x}, \mathbf{z}^{\prime}\right) \in S_{p, q-1}\left(d_{q-1} \mathbf{y}^{\prime}\right)$, the geometric simplex $\mathbf{z}^{\prime \prime} \in \Delta_{p+q+1} \mathcal{C}^{\prime}$ is defined as follows:

$$
z_{i}^{\prime \prime}=\left\{\begin{array}{ll}
z_{\sigma_{q-1}(i)}^{\prime} & i \neq q-1 \\
y_{q-1}^{\prime} & i=q-1
\end{array}, z_{i, j}^{\prime \prime}= \begin{cases}z_{\sigma_{q-1}(i), \sigma_{q-1}(j)}^{\prime} & q-1 \notin\{i, j\} \\
y_{i, q-1}^{\prime} & i \leq q-1=j \\
z_{q-1, j-1}^{\prime} y_{q-1, q}^{\prime} & i=q-1<j\end{cases}\right.
$$

and

$$
z_{i, j, k}^{\prime \prime}= \begin{cases}z_{\sigma_{q-1}(i), \sigma_{q-1}(j), \sigma_{q-1}(k)}^{\prime} & q-1 \notin\{i, j, k\} \\ y_{i, j, q-1}^{\prime} & k=q-1 \\ \left(z_{q-1, k-1}^{\prime} y_{i, q-1, q}^{\prime}\right) \circ z_{i, q-1, k-1}^{\prime} & j=q-1 \\ z_{q-1, j-1, k-1}^{\prime} y_{q-1, q}^{\prime} & i=q-1 .\end{cases}
$$

It is straightforward to check that:

- $d_{0}^{q+1} \mathbf{z}^{\prime \prime}=F(\mathbf{x})$ and $d_{q+1}^{p+1} \mathbf{z}^{\prime \prime}=\mathbf{y}^{\prime}$, so that $\left(\mathbf{x}, \mathbf{z}^{\prime \prime}\right) \in S_{p, q}\left(\mathbf{y}^{\prime}\right)$,

- $d_{q-1} \mathbf{z}^{\prime \prime}=\mathbf{z}^{\prime}$, whence $d_{q-1}^{v} \Gamma=I d$, and

- $\quad \Gamma$ is a simplicial map.

Finally, a simplicial homotopy $H: I d \Rightarrow \Gamma d_{q-1}^{v}$ is given by the maps

$$
H_{m}: S_{p, q}\left(\mathbf{y}^{\prime}\right) \longrightarrow S_{p+1, q}\left(\mathbf{y}^{\prime}\right), \quad\left(\mathbf{x}, \mathbf{z}^{\prime}\right) \mapsto\left(s_{m} \mathbf{x}, h_{m} \mathbf{z}^{\prime}\right), \quad 0 \leq m \leq p,
$$

where the geometric simplex $h_{m} \mathbf{z}^{\prime} \in \Delta_{p+q+1} \mathcal{C}^{\prime}$ is defined as $s_{q+m+1} \mathbf{z}^{\prime}=$ $\sigma_{q+m+1}^{*} \mathbf{z}^{\prime}$, except for the following elements:

$$
\begin{array}{ll}
\left(h_{m} \mathbf{z}^{\prime}\right)_{q-1, j}=z_{q, j}^{\prime} z_{q-1, q}^{\prime} & q<j \leq q+m+1, \\
\left(h_{m} \mathbf{z}^{\prime}\right)_{i, q-1, k}=\left(z_{q-1, q, k}^{\prime} z_{i, q-1}^{\prime}\right) \circ z_{i, q-1, k}^{\prime} & q<k \leq q+m+1, \\
\left(h_{m} \mathbf{z}^{\prime}\right)_{q-1, j, k}=z_{q, j, k}^{\prime} z_{q-1, q}^{\prime} & q \leq j \leq k \leq q+m+1, \\
\left(h_{m} \mathbf{z}^{\prime}\right)_{q-1, j, k}=\left(z_{q-1, j, k-1}^{\prime} z_{q-1, q}^{\prime}\right) \circ z_{q-1, q, k-1}^{\prime} & q<j \leq q+m+1<k .
\end{array}
$$


An iterative use of the above Lemma 3.2 shows that the composite simplicial map $\left(d_{1}^{v}\right)^{q}: S_{*, q}\left(\mathbf{y}^{\prime}\right) \longrightarrow S_{*, 0}\left(d_{1}^{q} \mathbf{y}^{\prime}\right)$ is a weak homotopy equivalence, for all $q \geq 1$ and all $\mathbf{y}^{\prime} \in \Delta_{q} \mathcal{C}^{\prime}$. But, for any object $y^{\prime} \in O b \mathcal{C}^{\prime}=\Delta_{0} \mathcal{C}^{\prime}$, we have that $S_{*, 0}\left(y^{\prime}\right) \cong \Delta\left(y^{\prime} / / F\right)$, and then

$$
\left|S_{*, 0}\left(y^{\prime}\right)\right| \cong\left|\Delta\left(y^{\prime} / / F\right)\right| \simeq \mathrm{B}\left(y^{\prime} / / F\right),
$$

where the latter is contractible by hypothesis. Hence, every $\left|S_{*, q}\left(\mathbf{y}^{\prime}\right)\right|$ is contractible.

It follows that each induced map $\left|\varphi_{q}^{\prime}\right|:\left|S_{*, q}\right| \rightarrow\left|\Delta_{q} \mathcal{C}^{\prime}\right|$ is a homotopy equivalence for all $q \geq 0$, and consequently so is the induced map $\left|\operatorname{diag} \varphi^{\prime}\right|:|\operatorname{diag} S| \rightarrow\left|\Delta \mathcal{C}^{\prime}\right|(21)$. This completes the proof of Theorem 2 .

We end this section by particularizing Theorem 2 to the case of a strict monoidal functor $F: \mathcal{C} \rightarrow \mathcal{C}^{\prime}$, between strict monoidal categories. To do so, recall that any monoidal category can be seen as a 2-category with only one object (see Remark at the end of Section 2).

Given such a strict monoidal functor $F: \mathcal{C} \rightarrow \mathcal{C}^{\prime}$, we shall define its "homotopy fiber 2-category", written by $\mathcal{K}_{F}$, as the homotopy fiber 2 -category of $F$ (considered as a 2-functor) at the unique object of $\mathcal{C}^{\prime}$. Then, $\mathcal{K}_{F}$ is the 2-category whose objects are the objects $y^{\prime}$ of $\mathcal{C}^{\prime}$, its arrows are pairs $\left(u^{\prime}, x\right): y^{\prime} \rightarrow z^{\prime}$ with $x$ an object in $\mathcal{C}$ and $u^{\prime}: z^{\prime} \rightarrow y^{\prime} \otimes F(x)$ an arrow in $\mathcal{C}^{\prime}$, and its deformations

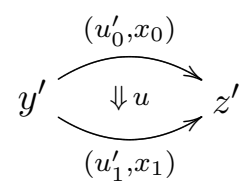

are those arrows $u: x_{0} \rightarrow x_{1}$ in $\mathcal{C}$ such that the following triangle commutes

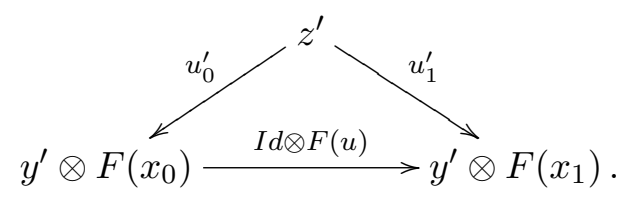

Then, as an immediate consequence of Theorem 2 and the Remark in Section 2, we have the following

COROLLARY 3.3. Let $F: \mathcal{C} \rightarrow \mathcal{C}^{\prime}$ be a strict monoidal functor between strict monoidal categories such that the classifying space $\mathrm{B} \mathcal{K}_{F}$ is contractible. Then, the induced map $\mathrm{BF}: \mathrm{BC} \rightarrow \mathrm{BC}^{\prime}$ is a homotopy equivalence. 


\section{References}

1. Artin, M and Mazur B.: On the Van Kampen Theorem, Topology 5 (1966), 179-189.

2. Borceux F.: Handbook of Categorical Algebra 1. Basic category theory, Cambridge Univ. Press, 1994.

3. Bousfield A. K. and Kan D. M.: Homotopy limits, completions and localizations, Lecture Notes in Math. 304, Springer-Verlag (1972).

4. Brown R. and Higgins P. J.: Sur les complexes croisś d'homotopie associés a quelques espaces filtrés, Comp. Rend. Acad. Sci. Paris 286 (1978), 91-93.

5. Duskin J.: A simplicial-matrix approach to higher dimensional category theory I: Nerves of bicategories, II: Nerves of morphisms of bicategories. preprints 2001.

6. Carrasco P. and Cegarra A. M.: (Braided) Tensor structures on homotopy groupoids and nerves of (braided) categorical groups, Communications in Algebra 24(13) (1996) 3995-4058.

7. Cegarra A. M. and Garzón A. R.: Homotopy classification of categorical torsors, Appl. Cat. Structures 9 (2001), 465-496.

8. Cordier J.-M.: Sur les limites homotopiques de diagrammes homotopiquement cohérents, Compositio Math. 62 (1987), 367-388.

9. Gray J. W.: Closed categories, lax limits and homotopy limits, Journal of Pure and Applied Algebra 19 (1980), 127-158.

10. Grothendieck A.: Théorie de la descente,... Séminaire Bourbaki 195 (19591960).

11. Illusie L.: Complexe cotangent et deformations II, Lecture Notes in Math. 283, Springer-Verlag (1972).

12. Hinich V. A. and Schechtman V. V.: Geometry of a category of complexes and algebraic K-theory, Duke Math. Journal 52 (1985), 339-430.

13. Quillen D.: Higher algebraic K-theory:I, in Algebraic K-theory I, Lecture Notes in Math. 341 (1973), 77-139.

14. Loday J.-L.: Spaces with finitely many homotopy groups, J. of Pure and Appl. Algebra 24 (1982), 179-202.

15. Mac Lane S.: Categories for the working mathematician, GTM 5 2nd Edition, Springer (1998).

16. May J.P.: Simplicial objects in Algebraic Topology, Van Nostrand ,1967.

17. May J.P.: The Geometry of Iterated Loop Spaces, Lecture Notes in Math. 271 (1972).

18. May J.P.: The spectra associated to permutative categories, Topology $\mathbf{1 7}$ (1979), 225-228.

19. Milnor J.: The geometrical realization of a semi-simplicial complex, Ann. of Math. 65 (1957), 357-362.

20. Moerdijk I. and Svensson J. A.: Algebraic classification of equivariant homotopy 2-types,I, J. of Pure and Appl. Algebra 89 (1993), 187-216.

21. Segal G.B.: Classifying spaces and spectral sequences, Publ. Math. Inst. des Hautes Etudes Scient. (Paris) 34 (1968), 105-112.

22. Segal G.B.: Categories and cohomology theories, Topology 13 (1974), 293-312.

23. Street R.: The algebra of oriented simplices, J. of Pure and Appl. Algebra 49(3) (1987), 283-335. 
Address for Offprints: Departamento de Álgebra Facultad de Ciencias, Universidad de Granada 18071 Granada

Spain 